\title{
DYNAMICS OF THREE MAGNONS \\ FOR THE DYSON HAMILTONIAN IN ONE DIMENSION
}

\author{
J.E. VAN HIMBERGEN and J.A. TJON \\ Instituut voor Theoretische Fysica der Rijksuniversiteit, \\ De Uithof, Utrecht, Nederland
}

Received 8 April 1974

\begin{abstract}
Synopsis
For a linear chain of $N$ spins $\frac{1}{2}$ with isotropic ferromagnetic exchange $(J>0)$ between nearest neighbours the complete spectrum of the Dyson hamiltonian is determined within the subspaces of boson occupation number $n=2$ and $n=3$. In addition to the three-magnon bound state at $E=\frac{1}{3} J(1-\cos K)$, there are also present three bound states which do not have their analogue in the Heisenberg chain. One of these states occurs at a real energy $E_{1}=(7 / 8) J$, while the other two are at complex-conjugate energies $E_{2}=(2.06+0.496 \mathrm{i}) J$ and $E_{3}=E_{2}^{*}$.

Using the calculated wave functions, the off-shell three-body $T$ matrix is determined near the bound-state energies. It is found that this $T$ matrix has a very simple analytic structure and it is explicitly verified that it is a solution of the Faddeev equation. Finally, the corresponding equations for the anisotropic case $\left(J_{x}=J_{y}=\sigma J, J_{z}=J\right)$ are briefly discussed.
\end{abstract}

1. Introduction. The problem of finding all eigenvalues and eigenvectors of the Heisenberg hamiltonian with nearest-neighbour interaction in one dimension was solved a long time ago by Bethe ${ }^{1}$ ). A frequently used boson representation of the Heisenberg hamiltonian is the so-called Dyson hamiltonian ${ }^{2}$ ). It is obtained from the Heisenberg hamiltonian by performing a Dyson-Maleev transformation to the spin operators ${ }^{3}$ ). The spectrum of the Dyson hamiltonian contains all the eigenvalues of the Heisenberg hamiltonian though the corresponding eigenstates are quite different. Moreover the spectrum is larger than that of the Heisenberg hamiltonian. This can be understood from the fact that boson occupation numbers higher than one (we restrict ourselves to spin $\frac{1}{2}$ ) can occur, so, since the occupation number of one site is the analogue of the number of spin deviations at that site, there is no restriction on the number of spin deviations at one site, which does exist in the real spin problem.

The Schrödinger equations resulting from the Dyson hamiltonian have never been solved analytically. Here we shall give the analytic solution for the cases $n=2$ and $n=3, n$ being the total number of "spin" deviations. This will give an idea of the spurious features introduced by using the Dyson hamiltonian instead 
of the Heisenberg hamiltonian. Indeed we shall find one unphysical bound state at $E=2 J$ for $n=2$ corresponding to two spin deviations at the same site, and one unphysical bound state at $E=0.875 J$ and an unphysical continuum of states with $2 J \leqslant E \leqslant 4 J$ for $n=3$. Moreover two solutions occur at complex energics $E_{1} \approx\left(2.06+0.496\right.$ i) $J$ and $E_{2}=E_{1}^{*}$ which in view of the nonhermiticity of the Dyson hamiltonian is not surprising. Here $J$ is the exchange integral, $J>0$, for we shall only consider the ferromagnetic case.

In particular, the presence of the unphysical bound state of three spin deviations warns us to be careful in using Dyson's hamiltonian instead of Heisenberg's hamiltonian when searching for bound-state properties of more complicated spin systems such as two- and three-dimensional Heisenberg spin systems. The formulation of the problem for these more complicated systems in terms of Dyson's hamiltonian may be simpler, but one could very well have introduced some spurious dynamical features that might not be recognizable as such because of the complexity of the system.

Another way of examining the dynamical properties of a physical system, is to study the $T$-matrix elements. As shown by Faddeev ${ }^{4}$ ) these elements satisfy for the case of $n=3$ a set of linear integral equations. These equations can in general not be solved analytically, and simple cases for which such an analytic solution is possible can contribute in understanding their structure. Dodd ${ }^{5}$ ) solved the Faddeev equations for a one-dimensional system of three particles with $\delta$-function interaction, which are otherwise free. In our case the Faddeev equations resulting from Dyson's hamiltonian can also be solved and it turns out that Dodd's assumptions for the kernel of the integral equation, which made an analytic solution possible, hold here too with a slight modification, in spite of the fact that the interaction and the dispersion relation of the free "particles" are quite different in our case.

An earlier attempt to treat the three-magnon problem of Dyson's hamiltonian by means of Faddeev equations was made by Majumdar ${ }^{6,7}$ ), but his result is hardly more than what one should consider as the formulation of the problem.

2. Formulation of the problem. Consider a linear chain of $N$ equidistant spins $\frac{1}{2}$ with distance $\Delta=1$ between neighbouring spins. The spins interact through nearest-neighbour ferromagnetic exchange $(J>0)$ :

$$
H_{\mathrm{s}}=-\frac{1}{2} J \sum_{\Delta= \pm 1} \sum_{i} s_{i} \cdot s_{i+\Delta}
$$

The operator $\bar{n}=\frac{1}{2} N+\sum_{i} s_{i}^{z}$ which gives the number of spin deviations from the totally aligned state $|0\rangle(\bar{n}=0)$ commutes with $H_{\mathrm{s}}$. One may therefore consider the problem of finding all eigenvalues and eigenvectors of $H_{\mathrm{s}}$ within a subspace of definite $\bar{n}$. For $H_{\mathrm{s}}$ this problem has been solved in general by Bethe ${ }^{1}$ ), who solved the Schrödinger equations resulting from $H_{\mathrm{s}}$ for arbitrary $\bar{n}$. 
In spite of the fact that the spin operators do not satisfy a definite boson or fermion statistics, the hamiltonian (2.1) is replaced by

$$
\begin{aligned}
H_{\mathrm{D}}= & \frac{1}{4} J \sum_{i} \sum_{\Delta= \pm 1} \\
& \times\left[\left(a_{i}^{+}-a_{i+\Delta}^{+}\right)\left(a_{i}-a_{i+\Delta}\right)+a_{i}^{+} a_{i+\Delta}^{+}\left(a_{i}-a_{i+\Delta}\right)^{2}\right],
\end{aligned}
$$

where $a_{i}^{+}, a_{i}$ are creation and annihilation operators of bosons at site $i$. This is the well-known Dyson hamiltonian ${ }^{2}$ ). Formally it can be obtained from eq. (2.1) by performing the Dyson-Maleev transformation ${ }^{3}$ ) to the spin operators $s_{i}$ : $s_{i}^{+}=a_{i}^{+}, s_{i}^{-}=a_{i}-a_{i}^{+} a_{i} a_{i}, s_{i}^{z}=-\frac{1}{2}+a_{i}^{+} a_{i}$. As is well known, this transformation is in accordance with the commutation rules for the spin operators $s_{i}$, but violates the hermitean character of $H_{\mathrm{s}}$. Furthermore the kinematic restriction on the number of spin deviations at one site is lost and this is felt most heavily in the case of $s=\frac{1}{2}$ that we are going to study here. Again the number operator $n=\sum_{i} a_{i}^{+} a_{i}$ commutes with $H_{\mathrm{D}}$, so we may consider the problem of finding all eigenvalues and eigenstates of $H_{\mathrm{D}}$ within subspaces of definite $n$. In sections 3,4 and 5 of this paper the solution to this problem is given for $n=2$ and $n=3$. Subsequently the bound-state solutions of three magnons will be used in section 6 to construct the explicit eigenfunctions of the Faddeev equations and it will be verified in section 7 that they are indeed solutions of the integral equations. Finally some concluding remarks are made in the last section.

3. Solution of the Schrödinger equations for two magnons. Let us consider the state with one spin deviation at site $m_{1}$ and one at site $m_{2}$. It is given by:

$$
\left|m_{1} m_{2}\right\rangle=a_{m_{1}}^{+} a_{m_{2}}^{+}|0\rangle
$$

where the vacuum state $|0\rangle$ is defined as $a_{i}|0\rangle=0^{*}$ for all $i$. We have as normalization condition:

$$
\left\langle n_{1} n_{2} \mid m_{1} m_{2}\right\rangle=\delta_{n_{1} m_{1}} \delta_{n_{2} m_{2}}+\delta_{n_{1} m_{2}} \delta_{n_{2} m_{1}}
$$

An arbitrary state $|\psi\rangle$ with $n=2$ is given by

$$
|\psi\rangle=\sum_{m_{1}, m_{2}=1}^{N} a_{m_{1} m_{2}}\left|m_{1} m_{2}\right\rangle .
$$

The Schrödinger equations for the amplitudes $a_{m_{1} m_{2}}$ are obtained by straightforward calculation of $\left\langle n_{1} n_{2}\left|H_{\mathrm{D}}\right| \psi\right\rangle=E\left\langle n_{1} n_{2} \mid \psi\right\rangle$ using (3.1) and the com- 
mutation rules for $a_{i}^{+}$and $a_{i}$. We arrive at the following equations:

$n_{1} \neq n_{2} \pm 1$ :

$$
E a_{n_{1} n_{2}}=2 J a_{n_{1} n_{2}}-\frac{1}{2} J\left(a_{n_{1}-1 n_{2}}+a_{n_{1}+1 n_{2}}+a_{n_{1} n_{2}-1}+a_{n_{1} n_{2}+1}\right),
$$

$n_{1}=n_{2}-1$ :

$$
E a_{n_{2}-1 n_{2}}=J a_{n_{2}-1 n_{2}}-\frac{1}{2} J\left(a_{n_{2}-2 n_{2}}+a_{n_{2}-1 n_{2}+1}\right),
$$

$n_{1}=n_{2}$ :

$$
E a_{n_{2} n_{2}}=2 J a_{n_{2} n_{2}}-J\left(a_{n_{2}-1 n_{2}}+a_{n_{2} n_{2}+1}\right) .
$$

The solution of these equations is particularly simple, but already exhibits the features needed in the more complicated case of $n=3$. In the following we set for convenience $J=1$ everywhere. First we note that (3.4a) and (3.4b) are identical to Bethe's equations. The latter lead to the well-known solution

$$
a_{n_{1} n_{2}}^{\mathbf{B}}=\mathrm{e}^{\mathrm{i}\left(k_{1} n_{1}+k_{2} n_{2}+\frac{1}{2} \varphi\right)}+\mathrm{e}^{\mathrm{i}\left(k_{2} n_{1}+k_{1} n_{2}-\frac{1}{2} \varphi\right)},
$$

where $n_{1}<n_{2}$ and $\varphi$ obeys

$$
\operatorname{cotg} \frac{1}{2} \varphi=\frac{\sin \tau}{\cos \frac{1}{2} K-\cos \tau}, \quad \tau=\frac{1}{2}\left(k_{1}-k_{2}\right) ; \quad K=k_{1}+k_{2} .
$$

For the momenta, it follows from the periodic boundary conditions that

$$
N k_{1}=2 \pi m_{1}+\varphi, \quad N k_{2}=2 \pi m_{2}-\varphi, \quad m_{1}, m_{2}=0,1, \ldots, N-1 .
$$

The energy eigenvalues in terms of $k_{1}$ and $k_{2}$ are

$$
E(K, \tau)=2-\cos k_{1}-\cos k_{2}=2\left(1-\cos \frac{1}{2} K \cos \tau\right) .
$$

In addition to the solutions of Bethe's equations with $k_{i}$ real, there is also for a given $K$ one solution with $k_{i}$ complex $k_{1}=k_{2}^{*}=\frac{1}{2} K+\mathrm{i} v$ and which corresponds to a two-magnon bound state. Its energy is given by $E=\frac{1}{2}(1-\cos K)$. For the details we refer to ref. 1.

Since (3.4a) and (3.4b) are identical to Bethe's equations these two equations alone are solved by

$$
a_{n_{1} n_{2}}=a_{n_{2} n_{1}}=a_{n_{i_{1} n_{i_{2}}}}^{\mathbf{B}}\left(k_{1}, k_{2}\right),
$$

where $\left(i_{1}, i_{2}\right)$ is that permutation of 1 and 2 such that $n_{i_{1}}<n_{i_{2}}$. For a given $E(K, \tau)$ from (3.8) the remaining amplitude $a_{n n}$ can be easily determined by 
eq. (3.4c). On substituting $a_{n-1 n}$ and $a_{n n+1}$ from (3.5), we find

$$
a_{n n}=\left(\cos \frac{1}{2} K / \cos \tau\right) \mathrm{e}^{1 K n} \cos \frac{1}{2} \varphi .
$$

Besides the above "physical" solutions we also have an additional solution which is given by $a_{n_{1} n_{2}}=0$ for $n_{1} \neq n_{2}$. Substituting this into eq. (3.4c) we get $a_{n n}$ $=$ constant $\neq 0$ provided $E=2$. This bound state is unphysical in the sense that there is no corresponding state in a Heisenberg chain of spins $\frac{1}{2}$. This completes the solution for the Dyson hamiltonian for $n=2$.

4. The physical states of three magnons. We now proceed to discuss the more complicated case of three magnons. An arbitrary state for $n=3$ can be written as

$$
|\psi\rangle=\sum_{m_{1}, m_{2}, m_{3}=1}^{N} a_{m_{1} m_{2} m_{3}}\left|m_{1} m_{2} m_{3}\right\rangle,
$$

where $\left|m_{1} m_{2} m_{3}\right\rangle$ corresponds to a state with spin deviations at sites $m_{1}, m_{2}$ and $m_{3}$

$$
\left|m_{1} m_{2} m_{3}\right\rangle=a_{m_{1}}^{+} a_{m_{2}}^{+} a_{m_{3}}^{+}|0\rangle
$$

Its normalization is given by

$$
\left\langle n_{1} n_{2} n_{3} \mid m_{1} m_{2} m_{3}\right\rangle=\sum_{\mathbf{P}\left(p_{i}\right)} \delta_{n_{p_{1}} m_{1}} \delta_{n_{p_{2}} m_{2}} \delta_{n_{p_{3}} m_{3}}
$$

where $\sum_{\mathbf{P}\left(p_{i}\right)}$ is a sum over all six permutations of $1,2,3$. Straightforward calculation of $\left\langle n_{1} n_{2} n_{3}\left|H_{\mathrm{D}}\right| \psi\right\rangle=E\left\langle n_{1} n_{2} n_{3} \mid \psi\right\rangle$ leads to the following equations for the amplitudes $a_{n_{1} n_{2} n_{3}}$ :

$n_{1}, n_{2}, n_{3}$ are no neighbours:

$$
\begin{aligned}
(E- & 3 J) a_{n_{1} n_{2} n_{3}} \\
= & -\frac{1}{2} J\left(a_{n_{1}+1 n_{2} n_{3}}+a_{n_{1}-1 n_{2} n_{3}}+a_{n_{1} n_{2}+1 n_{3}}+a_{n_{1} n_{2}-1 n_{3}}\right. \\
& \left.+a_{n_{1} n_{2} n_{3}+1}+a_{n_{1} n_{2} n_{3}-1}\right)
\end{aligned}
$$

$n_{2}=n_{1}+1, n_{3}$ no neighbour of $n_{1}$ or $n_{2}$ :

$$
\begin{aligned}
& (E-2 J) a_{n_{1} n_{1}+1 n_{3}} \\
& \quad=-\frac{1}{2} J\left(a_{n_{1}-1 n_{1}+1 n_{3}}+a_{n_{1} n_{1}+2 n_{3}}+a_{n_{1} n_{1}+1 n_{3}+1}+a_{n_{1} n_{1}+1 n_{3}-1}\right)
\end{aligned}
$$

$n_{2}=n_{1}+1, n_{3}=n_{1}+2:$

$$
(E-J) a_{n_{1} n_{1}+1 n_{1}+2}=-\frac{1}{2} J\left(a_{n_{1}-1 n_{1}+1 n_{1}+2}+a_{n_{1} n_{1}+1 n_{1}+3}\right)
$$




$$
\begin{aligned}
& n_{1}=n_{2}, n_{3}>n_{1}+1: \\
& (E-3 J) a_{n_{1} n_{1} n_{3}} \\
& \quad=-J\left(a_{n_{1}-1 n_{1} n_{3}}+a_{n_{1} n_{1}+1 n_{3}}+\frac{1}{2} a_{n_{1} n_{1} n_{3}-1}+\frac{1}{2} a_{n_{1} n_{1} n_{3}+i}\right)
\end{aligned}
$$

$n_{1}=n_{2}, n_{3}=n_{1}+1:$

$$
(E-J) a_{n_{1} n_{1} n_{1}+1}=\frac{1}{2} J\left(a_{n_{1} n_{1} n_{1}}-a_{n_{1} n_{1} n_{1}+2}-2 a_{n_{1}-1 n_{1} n_{1}+1}\right) ;
$$

$n_{1}=n_{2}=n_{3}$

$$
(E-3 J) a_{n_{1} n_{1} n_{1}}=-\frac{3}{2} J\left(a_{n_{1}-1 n_{1} n_{1}}+a_{n_{1} n_{1} n_{1}+1}\right)
$$

$n_{1}=n_{2}, n_{3}=n_{1}-1:$

$$
(E-J) a_{n_{1}-1 n_{1} n_{1}}=\frac{1}{2} J\left(a_{n_{1} n_{1} n_{1}}-a_{n_{1}-2 n_{1} n_{1}}-2 a_{n_{1}-1 n_{1} n_{1}+1}\right)
$$

$n_{1}=n_{2}, n_{3}<n_{1}-1:$

$$
\begin{aligned}
(E & -3 J) a_{n_{3} n_{1} n_{1}} \\
& =-J\left(a_{n_{3} n_{1}-1 n_{1}}+a_{n_{3} n_{1} n_{1}+1}+\frac{1}{2} a_{n_{3}-1 n_{1} n_{1}}+\frac{1}{2} a_{n_{3}+1 n_{1} n_{1}}\right) .
\end{aligned}
$$

Eqs. (4.4a)-(4.4c) are identical to Bethe's equations. Similarly as in the case of $n=2$, these equations alone can then be solved by

$$
a_{n_{p_{1}} n_{p_{2}} n_{p_{3}}}=a_{n_{i_{1} n_{2} n_{i_{3}}}^{\mathrm{B}}}^{\mathrm{B}}\left(k_{1}, k_{2}, k_{3}\right)
$$

where $\left(p_{1}, p_{2}, p_{3}\right)$ is any permutation of $1,2,3$ and $i_{1}, i_{2}, i_{3}$ is that permutation of $1,2,3$ for which $n_{i_{1}}<n_{i_{2}}<n_{i_{3}} . a^{\mathbf{B}}$ is again the Bethe amplitude given by

$$
\begin{aligned}
& a_{n_{i_{1}} n_{i_{2}} n_{i_{3}}}^{\mathbf{B}}\left(k_{1}, k_{2}, k_{3}\right) \\
& \quad=\sum_{\mathbf{P}(p)} \exp \left[\mathrm{i}\left(k_{p_{1}} n_{i_{1}}+k_{p_{2}} n_{i_{2}}+k_{p_{3}} n_{i_{3}}+\frac{1}{2} \sum_{k<l} \varphi_{p_{k} p_{l}}\right)\right],
\end{aligned}
$$

with $\varphi_{p_{k} p_{l}}$ satisfying

$$
\operatorname{cotg} \frac{1}{2} \varphi_{n}=\sin p_{n} /\left(\cos \frac{1}{2} K_{n}-\cos p_{n}\right) \quad(n=1,2,3) .
$$

Here we have defined $\varphi_{1} \equiv \varphi_{23}, \varphi_{2} \equiv \varphi_{13}, \varphi_{3} \equiv \varphi_{12}$ and $p_{1}=\frac{1}{2}\left(k_{2}-k_{3}\right)$, $K_{1}=k_{2}+k_{3}, p_{2}=\frac{1}{2}\left(k_{1}-k_{3}\right), K_{2}=k_{1}+k_{3}, p_{3}=\frac{1}{2}\left(k_{1}-k_{2}\right), K_{3}=k_{1}+k_{2}$. 
The energy eigenvalue can be expressed in terms of the above momenta:

$$
\begin{aligned}
E\left(k_{1}, k_{2}, k_{3}\right) & =\sum_{i=1}^{3}\left(1-\cos k_{i}\right) \\
& =3-\cos k_{n}-2 \cos \frac{1}{2} K_{n} \cos p_{n} \quad(n=1,2,3) .
\end{aligned}
$$

The remaining equations (4.5) can now be solved for a given $E\left(k_{1}, k_{2}, k_{3}\right)$ by writing for the unknown amplitudes in (4.5):

$$
a_{n_{1} n_{1} n_{3}}=2 \sum_{m=1}^{3} c_{n_{3}}^{m}\left(n_{1}\right) \exp \left(\mathrm{i} k_{m} n_{3}+\mathrm{i} K_{m} n_{1}+\mathrm{i} \chi_{m}\right) \cos \frac{1}{2} \varphi_{m},
$$

where $\chi_{1}=-\frac{1}{2}\left(\varphi_{2}+\varphi_{3}\right), \chi_{2}=\frac{1}{2}\left(\varphi_{3}-\varphi_{1}\right), \chi_{3}=\frac{1}{2}\left(\varphi_{1}+\varphi_{2}\right)$. Inserting (4.10). and (4.7) into (4.5) yields the equations:

$$
\begin{aligned}
& a c_{n_{3}}^{m}\left(n_{1}\right)+\frac{1}{2}\left[\mathrm{e}^{-1 k_{m}} c_{n_{3}-1}^{m}\left(n_{1}\right)+\mathrm{e}^{\mathrm{i} k_{m}} c_{n_{3}+1}^{m}\left(n_{1}\right)\right]=-2 \cos ^{2} \frac{1}{2} K_{m} \\
& b \mathrm{e}^{\mathrm{i} k_{m}} c_{n_{1}+1}^{m}\left(n_{1}\right)+\frac{1}{2}\left[\mathrm{e}^{2 \mathrm{i} k_{m}} c_{n_{1}+2}^{m}\left(n_{1}\right)-c_{n_{1}}^{m}\left(n_{1}\right)\right]=-\mathrm{e}^{\mathrm{i} k_{m}-\mathrm{i} K_{m} / 2} \cos \frac{1}{2} K_{m} \\
& a c_{n_{1}}^{m}\left(n_{1}\right)+\frac{3}{2}\left[\mathrm{e}^{-\mathrm{i} k_{m}} c_{n_{1}-1}^{m}\left(n_{1}\right)+\mathrm{e}^{\mathrm{i} k_{m}} c_{n_{1}+1}^{m}\left(n_{1}\right)\right]=0 \\
& b \mathrm{e}^{-\mathrm{i} k_{m}} c_{n_{1}-1}^{m}\left(n_{1}\right)+\frac{1}{2}\left[\mathrm{e}^{-2 \mathrm{i} k_{m}} c_{n_{1}-2}^{m}\left(n_{1}\right)-c_{n_{1}}^{m}\left(n_{1}\right)\right] \\
& \quad=-\mathrm{e}^{-\mathrm{i} k_{m}+\frac{1}{2} \mathrm{i} K_{m}-2 \mathrm{i} \chi_{m}} \cos \frac{1}{2} K_{m} \\
& a c_{n_{3}}^{m}\left(n_{1}\right)+\frac{1}{2}\left[\mathrm{e}^{-1 k_{m}} c_{n_{3}-1}^{m}\left(n_{1}\right)+\mathrm{e}^{\mathrm{i} k_{m}} c_{n_{3}+1}^{m}\left(n_{1}\right)\right]=-2 \cos ^{2} \frac{1}{2} K_{m} \mathrm{e}^{-2 \mathrm{i} \chi_{m}},
\end{aligned}
$$

where $a \equiv E-3$ and $b \equiv E-1$.

From (4.11) we may now determine the $n_{3}$ dependence of $c_{n_{3}}^{m}\left(n_{1}\right)$, which we denote for convenience by $c_{n_{3}}$. Let us write $c_{n_{3}}$ as

$$
c_{n_{3}}=s_{n_{3}}+e_{n_{3}},
$$

with

$$
\begin{aligned}
& s_{n_{3}}=\cos \frac{1}{2} K_{m} / \cos p_{m}, \quad n_{3}>n_{1}, \\
& s_{n_{3}}=\left(\cos \frac{1}{2} K_{m} / \cos p_{m}\right) \mathrm{e}^{-21 x_{m}}, \quad n_{3}<n_{1}, \\
& s_{n_{1}}=0 .
\end{aligned}
$$

Note that $s_{n_{3}}$ is a particular solution of (4.11a) and (4.11e). Therefore one obtains on substitution of (4.12) and (4.13) into (4.11)

$$
a e_{n_{3}}+\frac{1}{2}\left(\mathrm{e}^{-i k_{m}} e_{n_{3}-1}+\mathrm{e}^{\mathrm{i} k_{m}} e_{n_{3}+1}\right)=0
$$




$$
\begin{aligned}
& b \mathrm{e}^{\mathrm{i} k_{m}} e_{n_{1}+1}+\frac{1}{2}\left(\mathrm{e}^{2 \mathrm{i} k_{m}} e_{n_{1}+2}-e_{n_{1}}\right) \\
& \quad=-\mathrm{e}^{\mathrm{i} k_{m}-\mathrm{i} K_{m} / 2} \cos \frac{1}{2} K_{m}-b \mathrm{e}^{i k_{m}} s_{n_{1}+1}-\frac{1}{2} \mathrm{e}^{2 \mathrm{i} k_{m}} s_{n_{1}+2}, \\
& a e_{n_{1}}+\frac{3}{2}\left(\mathrm{e}^{-\mathrm{i} k_{m}} e_{n_{1}-1}+\mathrm{e}^{\mathrm{i} k_{m}} e_{n_{1}+1}\right)=-\frac{3}{2}\left(\mathrm{e}^{-\mathrm{i} k_{m}} s_{n_{1}-1}+\mathrm{e}^{\mathrm{i} k_{m}} S_{n_{1}+1}\right), \\
& b \mathrm{e}^{-\mathrm{i} k_{m}} e_{n_{1}-1}+\frac{1}{2}\left(\mathrm{e}^{-2 \mathrm{i} k_{m}} e_{n_{1}-2}-e_{n_{1}}\right) \\
& \quad=-\mathrm{e}^{-\mathrm{i} k_{m}+\frac{1}{2} \mathrm{i} K_{m}-2 \mathrm{i} \chi_{m}} \cos \frac{1}{2} K_{m}-b \mathrm{e}^{-\mathrm{i} k_{m}} s_{n_{1}-1}-\frac{1}{2} \mathrm{e}^{-2 \mathrm{i} k_{m}} s_{n_{1}-2}, \\
& a e_{n_{3}}+\frac{1}{2}\left(\mathrm{e}^{-\mathrm{i} k_{m}} e_{n_{3}-1}+\mathrm{e}^{\mathrm{i} k_{m}} e_{n_{3}+1}\right)=0 .
\end{aligned}
$$

Renumbering the lattice sites as follows

$$
\begin{aligned}
& n_{1}+2, n_{1}+3, \ldots, N \rightarrow 1,2, \ldots, N-n_{1}-1, \\
& 1,2, \ldots, n_{1}-2 \rightarrow N-n_{1}, N-n_{1}+1, \ldots, N-3, \\
& n_{1}-1, n_{1}, n_{1}+1 \rightarrow N-2, N-1, N,
\end{aligned}
$$

one obtains the following set of equations:

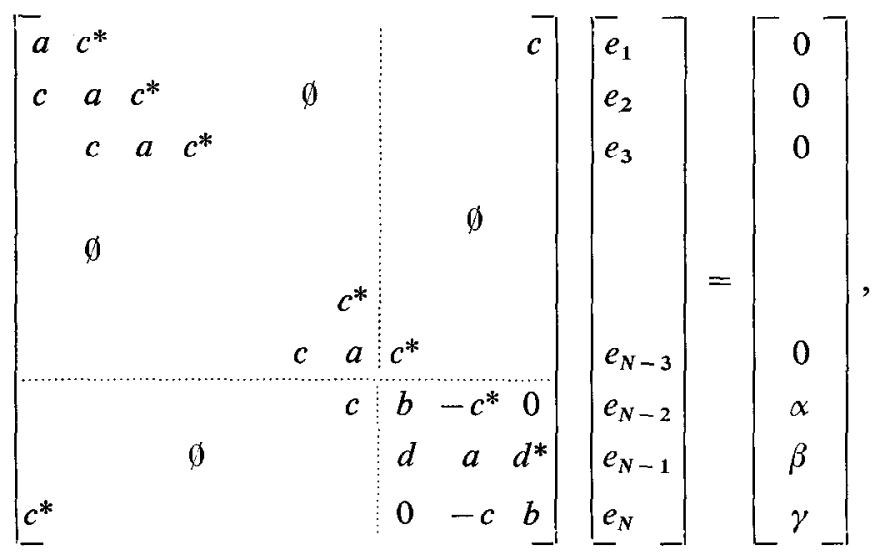

where $c=\frac{1}{2} \mathrm{e}^{-\mathrm{i} k_{m}}, c^{*}=\frac{1}{2} \mathrm{e}^{+\mathrm{i} k_{m}}, d=\frac{3}{2} \mathrm{e}^{-\mathrm{i} k_{m}}, d^{*}=\frac{3}{2} \mathrm{e}^{+1 k_{m}}$,

$$
\begin{aligned}
& \alpha=-\mathrm{e}^{\frac{1}{2} \mathrm{i} K_{m}-2 \mathrm{i} \chi_{m}} \cos \frac{1}{2} K_{m}-b s_{n_{1}-1}-\frac{1}{2} \mathrm{e}^{-\mathrm{i} k_{m}} s_{n_{1}-2}, \\
& \beta=-\frac{3}{2} \mathrm{e}^{-\mathrm{i} k_{m}} s_{n_{1}-1}-\frac{3}{2} \mathrm{e}^{\mathrm{i} k_{m}} s_{n_{1}+1}, \\
& \gamma=-\mathrm{e}^{-\mathrm{i} K_{m} / 2} \cos \frac{1}{2} K_{m}-b s_{n_{1}+1}-\frac{1}{2} \mathrm{e}^{\mathrm{i} k_{m}} s_{n_{1}+2} .
\end{aligned}
$$

The elements $E_{1 N}=c$ and $E_{N 1}=c^{*}$ take care of periodic boundary conditions.

The advantage of singling out the special solution $s_{n_{3}}$ is clear: the inhomogeneous term is only nonzero for the last three elements, which makes (4.15) more tractable.

The solution of (4.15) is given in appendix A. Upon substitution of (4.12) into (4.10) where $s_{n_{3}}$ and $e_{n_{3}}$ are given by (4.13) and the solution of (4.15) respectively, 
we find the amplitudes $a_{n_{1} n_{1} n_{3}}$. They determine together with the amplitudes $a_{n_{1} n_{2} n_{3}}$ given by (4.6) the complete wave function of the scattering states of three free magnons with momenta $k_{1}, k_{2}, k_{3}$ at energy $\sum_{i=1}^{3}\left(1-\cos k_{i}\right)$.

The Heisenberg chain has also one bound state of three magnons at energy $E_{\mathrm{p}}=\frac{1}{3}(1-\cos K)$. The Dyson chain has a bound state at the same energy. Since we shall be concerned later on with this solution, we now determine its wave function.

As in the case of scattering states, all amplitudes $a_{n_{1} n_{2} n_{3}}\left(n_{1} \neq n_{2} \neq n_{3} \neq n_{1}\right)$ are given by (4.6), and $a_{n_{i_{1}} n_{i_{2}} n_{i_{3}}}^{\mathbf{B}}$ are given by (4.7). However, a considerable simplification occurs in (4.7), in the limit $N \rightarrow \infty$ only one term in $\sum_{\mathbf{P}(p)}$ is left. According to Bethe we have

$$
a_{n_{i_{1}} n_{i_{2}} n_{i_{3}}}^{\mathrm{P}}=\mathrm{e}^{\mathrm{i} k_{2} n_{i_{2}}+\mathrm{i} u\left(n_{i_{1}}+n_{i_{3}}\right)} \mathrm{e}^{-v\left(n_{i_{3}}-n_{i_{1}}\right)} \mathrm{e}^{v N}, \quad n_{i_{1}}<n_{i_{2}}<n_{i_{3}},
$$

(the dependence on $N$ of all other terms in $\sum_{\mathbf{P}(p)}$ ranges from $\mathrm{e}^{v N / 2}$ to $\mathrm{e}^{-v N}$ ). The reason is that some of the phase factors $\varphi_{i}$ have an imaginary part proportional to $N$ for the bound state: $\operatorname{Im} \varphi_{1}=\operatorname{Im} \varphi_{3}=v N$, while $\varphi_{2}$ is finite. As a result (4.16) may easily be identified as the leading term of (4.7). The parameters $k_{2}$, $u$ and $v$ are defined as follows:

$$
\begin{aligned}
& k_{1}=u+\mathrm{i} v ; \quad k_{2}=K-2 u ; \quad k_{3}=u-\mathrm{i} v, \\
& z=3 \operatorname{cotg} K, \quad u=\operatorname{arccotg}(z / 3)-\operatorname{arccotg} z, \\
& \mathrm{e}^{-2 v}=\left(1+z^{2}\right) /\left(9+z^{2}\right) .
\end{aligned}
$$

With the above solution we proceed to determine the unphysical amplitudes. Similarly as in the case of the scattering states we write:

$$
\begin{aligned}
n_{3} \geqslant & n_{1}+1: \\
& a_{n_{1} n_{1} n_{3}}=\mathrm{e}^{N v} \mathrm{e}^{\mathrm{i} k_{2} n_{1}+\mathrm{i} u\left(n_{1}+n_{3}\right)} \mathrm{e}^{-v\left(n_{3}-n_{1}\right)} c_{>}+a_{n_{1} n_{1} n_{3}}^{\mathrm{E}} \\
n_{3}= & n_{1}: \\
& a_{n_{1} n_{1} n_{1}}=a_{n_{1} n_{1} n_{1}}^{\mathrm{E}} ; \\
n_{3} \leqslant & n_{1}-1: \\
& a_{n_{3} n_{1} n_{1}}=\mathrm{e}^{N v} \mathrm{e}^{\mathrm{i} k_{2} n_{1}+\mathrm{i} u\left(n_{1}+n_{3}\right)} \mathrm{e}^{-v\left(n_{1}-n_{3}\right)} c_{<}+a_{n_{3} n_{1} n_{1}}^{\mathrm{E}} .
\end{aligned}
$$

Choosing

$$
\begin{aligned}
& c_{>}=\frac{\mathrm{e}^{-\mathrm{i} u-v}+\mathrm{e}^{\mathrm{i} k_{2}}}{\cos (u-\mathrm{i} v)+\cos k_{2}}, \\
& c_{<}=\frac{\mathrm{e}^{\mathrm{iu-v}+\mathrm{e}^{-\mathrm{i} k_{2}}}}{\cos (u+\mathrm{i} v)+\cos k_{2}},
\end{aligned}
$$


one finds the following equations for $a_{n_{1} n_{1} n_{3}}^{\mathrm{E}}$ :

$$
\begin{aligned}
& a a_{n_{1} n_{1} n_{3}}^{\mathrm{E}}+\frac{1}{2}\left(a_{n_{1} n_{1} n_{3}-1}^{\mathrm{E}}+a_{n_{1} n_{1} n_{3}+1}^{\mathrm{E}}\right)=0, \\
& \frac{1}{2} a_{n_{1}-2 n_{1} n_{1}}^{\mathrm{E}}+b a_{n_{1}-1 n_{1} n_{1}}^{\mathrm{E}}-\frac{1}{2} a_{n_{1} n_{1} n_{1}}^{\mathrm{E}}=\alpha, \\
& \frac{3}{2} a_{n_{1}-1 n_{1} n_{1}}^{\mathrm{E}}+a a_{n_{1} n_{1} n_{1}}^{\mathrm{E}}+\frac{3}{2} a_{n_{1} n_{1} n_{1}+1}^{\mathrm{E}}=\beta, \\
& -\frac{1}{2} a_{n_{1} n_{1} n_{1}}^{\mathrm{E}}+b a_{n_{1} n_{1} n_{1}+1}^{\mathrm{E}}+\frac{1}{2} a_{n_{1} n_{1} n_{1}+2}^{\mathrm{E}}=\gamma, \\
& a a_{n_{3} n_{1} n_{1}}^{\mathrm{E}}+\frac{1}{2}\left(a_{n_{3}-1 n_{1} n_{1}}^{\mathrm{E}}+a_{n_{3}+1 n_{1} n_{1}}^{\mathrm{E}}\right)=0,
\end{aligned}
$$

where

$$
\begin{aligned}
& \alpha=-a_{n_{1}-1 n_{1} n_{1}+1}^{\mathrm{B}}-b a_{n_{1}-1 n_{1} n_{1}}^{\mathrm{S}}-\frac{1}{2} a_{n_{1}-2 n_{1} n_{1}}^{\mathrm{S}}, \\
& \beta=-\frac{3}{2}\left(a_{n_{1}-1 n_{1} n_{1}}^{\mathrm{S}}+a_{n_{1} n_{1} n_{1}+1}^{\mathrm{S}}\right), \\
& \gamma=-a_{n_{1}-1 n_{1} n_{1}+1}^{\mathrm{S}}-b a_{n_{1} n_{1} n_{1}+1}^{\mathrm{S}}-\frac{1}{2} a_{n_{1} n_{1} n_{1}+2}^{\mathrm{S}} .
\end{aligned}
$$

[The superscript S refers to the terms with $c_{<}$and $c_{>}$in (4.18).] Writing $a_{n_{1} n_{1} n_{3}}^{\mathrm{E}}$ in the form:

$$
a_{n_{1} n_{1} n_{3}}^{\mathrm{E}}=\mathrm{e}^{N v} \mathrm{e}^{1 K n_{1}} e_{n_{3}}\left(n_{1}\right)
$$

and renumbering the points in the chain as before, one obtains:

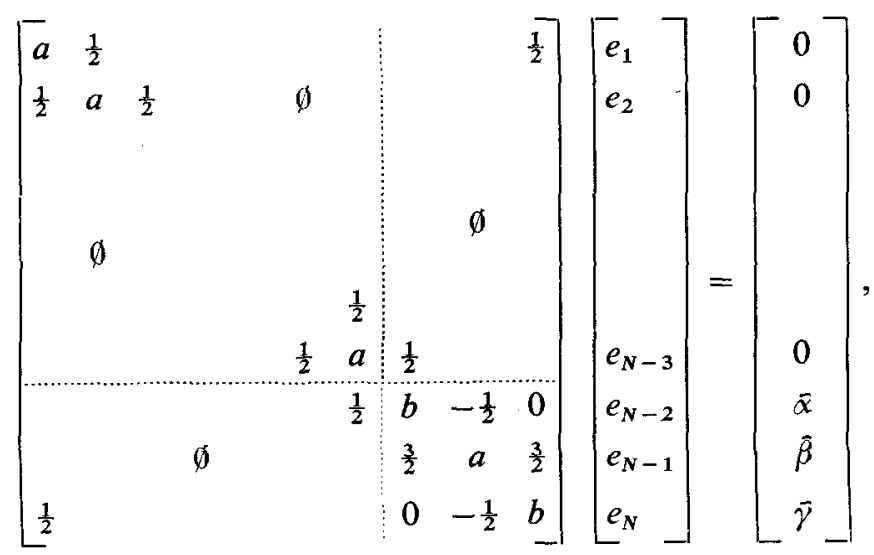

with

$$
\left(\begin{array}{l}
\bar{\alpha} \\
\bar{\beta} \\
\bar{\gamma}
\end{array}\right)=\mathrm{e}^{-\mathrm{i} K n_{1}} \mathrm{e}^{-v N}\left(\begin{array}{l}
\alpha \\
\beta \\
\gamma
\end{array}\right) .
$$


The solution of (4.22) is given in appendix $\mathrm{A}\left(k_{n}=0\right)$. Note that for all values of $K-3 \leqslant a \leqslant-\frac{7}{3}$, so that we have from (A.7)

$$
\begin{aligned}
& e_{n_{1}+\mu}=\left[\left(a^{2}-1\right)^{\frac{1}{2}}-a\right]\left|\left(a^{2}-1\right)^{\frac{1}{2}}+a\right|^{\mu} e_{n_{1}+1}, \\
& e_{n_{1}-\mu}=\left[\left(a^{2}-1\right)^{\frac{1}{2}}-a\right]\left|\left(a^{2}-1\right)^{\frac{1}{2}}+a\right|^{\mu} e_{n_{1}-1} .
\end{aligned}
$$

Since $\left|\left(a^{2}-1\right)^{\frac{1}{2}}+a\right|<1, e_{n_{1} \pm \mu} \rightarrow 0$ if $\mu \rightarrow \infty$, as it should for a bound state.

In the spin space corresponding to the Heisenberg chain there are $\frac{1}{6} N(N-1)$ $\times(N-2)$ configurations of three spin deviations, hence $\frac{1}{6} N(N-1)(N-2)$ independent eigenstates of the Heisenberg hamiltonian. Bethe determined all of these states. It should be noted that in addition to the scattering states of three magnons discussed here in detail, there are also other states, found by Bethe, which describe the scattering of a magnon on a bound-state complex of two magnons. These states can be discussed in precisely the same way as above.

In the oscillator space corresponding to the Dyson chain there are $\frac{1}{6} N(N-1)$ $\times(N-2)+N(N-1)+N$ configurations of three "spin" deviations, hence $\frac{1}{6} N(N-1)(N-2)+N^{2}$ independent eigenstates of the Dyson hamiltonian. We showed explicitly that the spectrum of $\frac{1}{6} N(N-1)(N-2)$ of these states coincides with the spectrum of the Heisenberg hamiltonian, and calculated the corresponding eigenstates. As a consequence a number of $N^{2}$ states remains to be determined for the Dyson hamiltonian. These states do not have their analogue in the Heisenberg problem and will be discussed in the next section.

5. Unphysical states of three magnons. We now investigate states $|\psi\rangle$ $=\sum_{n_{1} n_{2} n_{3}} a_{n_{1} n_{2} n_{3}}\left|n_{1} n_{2} n_{3}\right\rangle$ in which all physical amplitudes are zero:

$$
a_{n_{1} n_{2} n_{3}}=0, \quad n_{1} \neq n_{2} \neq n_{3} \neq n_{1} \text {. }
$$

We denote $a_{n_{1} n_{1} n_{3}}$ as $c_{n_{3}}\left(n_{1}\right)$. To investigate the solutions, the sites are renumbered as before. By virtue of (5.1) eqs. (4.5) then lead to a set of homogeneous equations for $c_{n_{3}}\left(n_{1}\right)$

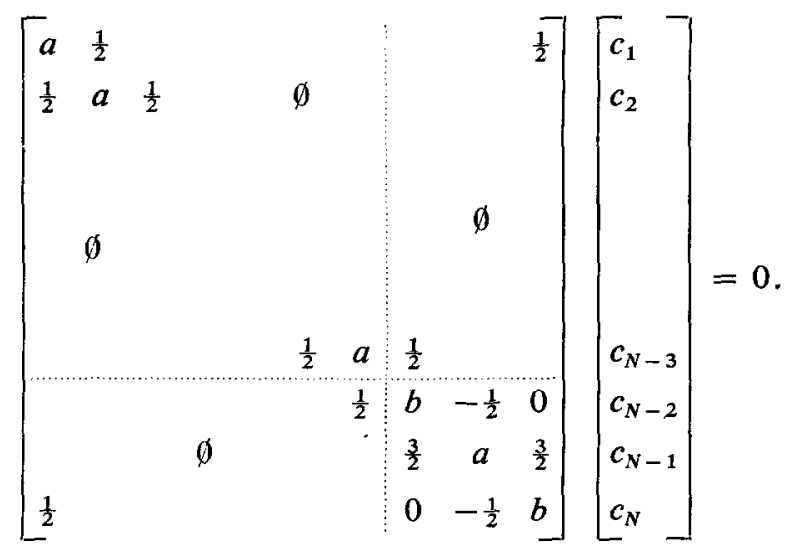

Eq. (5.2) has been studied in appendix A. 
According to eqs. (A.4) we have for finite $N$ and for $k_{n}=0$

$$
\begin{aligned}
& X_{v}=-\frac{1}{2} \lambda_{v}(E)^{-1}\left(\varphi_{N-3}^{v} c_{N-2}+\varphi_{1}^{v} c_{N}\right), \\
& \frac{1}{2} \sum_{\mu=1}^{N-3} \phi_{N-3}^{\mu} X_{\mu}+b c_{N-2}-\frac{1}{2} c_{N-1}=0, \\
& \frac{1}{2} \sum_{\mu=1}^{N-3} \phi_{1}^{\mu} X_{\mu}-\frac{1}{2} c_{N-1}+b c_{N}=0, \\
& \frac{3}{2} c_{N-2}+a c_{N-1}+\frac{3}{2} c_{N}=0,
\end{aligned}
$$

where

$$
X_{v}=\sum_{i=1}^{N-3} \phi_{i}^{v} c_{i} .
$$

Substraction of (5.4b) from (5.4a) and substitution of (5.3) into the result yields:

$$
(b-\Gamma+\Delta)\left(c_{N-2}-c_{N}\right)-0 .
$$

We now distinguish between two different types of solutions namely

$$
b-\Gamma+\Delta=0
$$

and $c_{N-2}=c_{N}$, which gives in view of eys. (5.4)

$$
a(-\Gamma+b-\Delta)+\frac{3}{2}=0 .
$$

In explicit form eqs. (5.7) and (5.8) read:

$$
b(E)-\Gamma_{\text {even }}(E)=0
$$

and

$$
a(E)\left(b(E)-\Gamma_{\text {odd }}(E)\right)+\frac{3}{2}=0,
$$

with

$$
\Gamma_{\text {even }}(E)=\frac{1}{2} \sum_{\substack{v=2 \\ v \text { even }}}^{N-3}\left|\varphi_{1}^{\nu}\right|^{2} / \lambda_{v}(E)
$$

and

$$
\Gamma_{\text {odd }}(E)=\frac{1}{2} \sum_{\substack{v=1 \\ v \text { odd }}}^{N-3}\left|\varphi_{1}^{v}\right|^{2} / \lambda_{v}(E) .
$$


These equations can be solved graphically for real values of $E$. In figs. 1 and 2 the results are given for $N=10$ and $N=12$, representing all typical choices of $N$. For $N=4,8,12,16, \ldots$ the solution $a=0$ is missing. As can be seen from (5.3) our derivation of (5.7) and (5.8) is not valid for this particular value of $a$, since
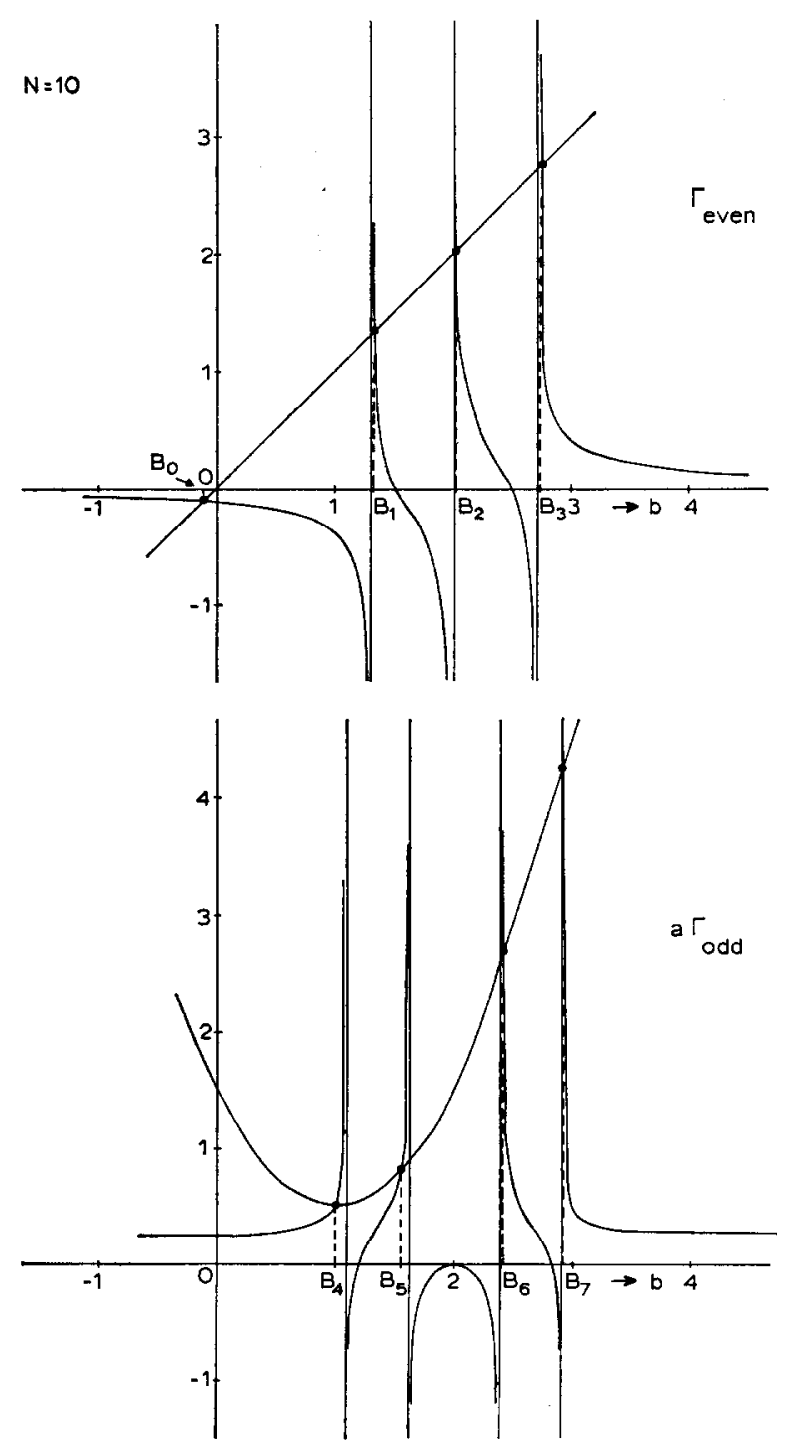

Fig. 1. Graphical solution of eqs. (5.7a) and (5.8a) for $N=10$. Solutions $B_{i}$ occur at the points of intersection of $b$ and $b(b-2)+\frac{3}{2}$ with $\Gamma_{\text {even }}$ and $a \Gamma_{\text {odd }}$, respectively. 
one divides by $\lambda_{\frac{1}{2}(N-2)}(3)=0$. The solution at $a=0$ can easily be obtained directly from eqs. (4.5):

$$
\begin{aligned}
& c_{n_{1}-1}\left(n_{1}\right)=c_{n_{1}+1}\left(n_{1}\right)=0, \quad c_{n_{1}}\left(n_{1}\right)=\Lambda_{0} \mathrm{e}^{\mathrm{i} K n_{1}}, \\
& c_{n_{1}+2 k}\left(n_{1}\right)=(-1)^{k+1} \Lambda_{0} \mathrm{e}^{\mathrm{i} K n_{1}}, \quad c_{n_{1}+2 k+1}\left(n_{1}\right)=0 .
\end{aligned}
$$
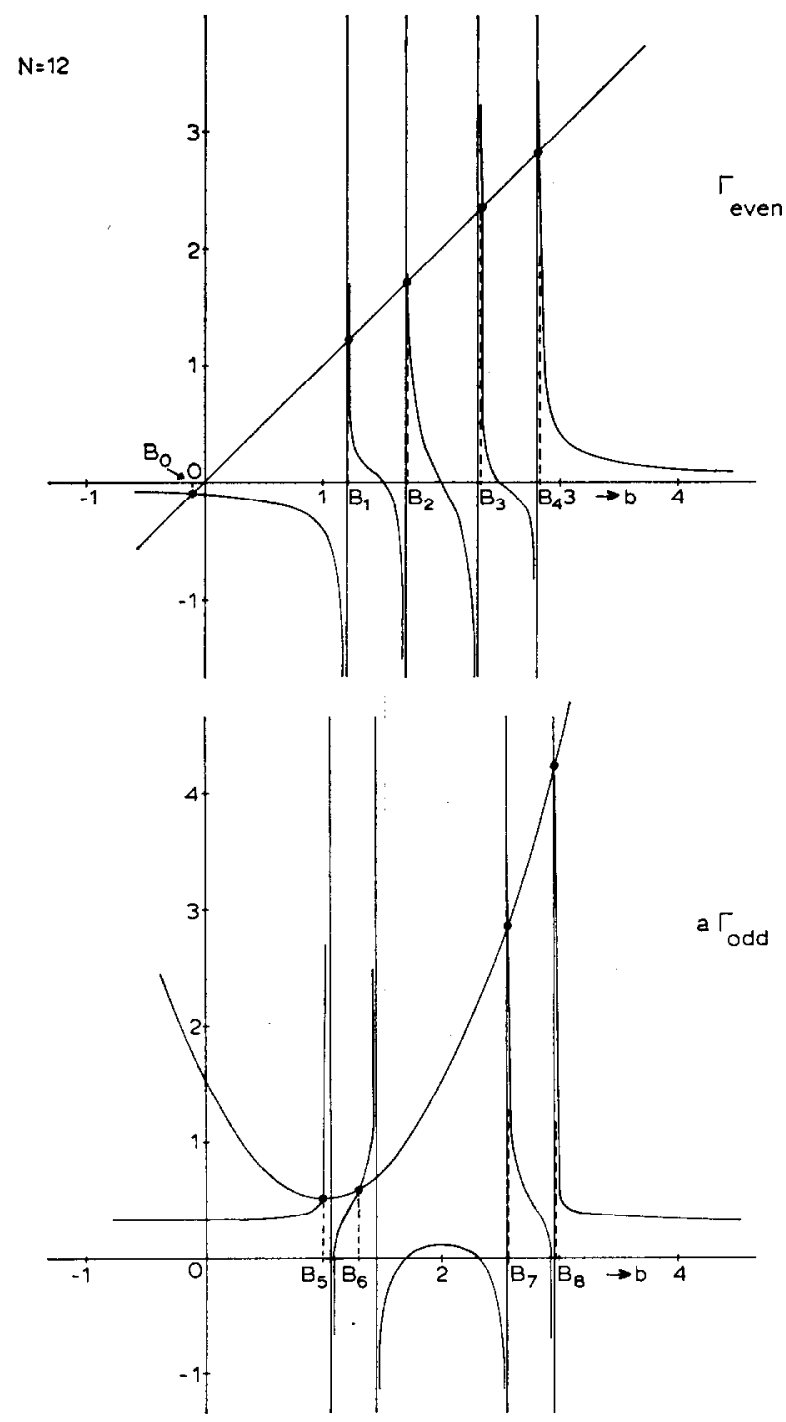

Fig. 2. Graphical solution of eqs. (5.7a) and (5.8a) for $N=12$. Solutions $B_{i}$ occur at the points of intersection of $b$ and $b(b-2)+\frac{3}{2}$ with $\Gamma_{\text {even }}$ and $a \Gamma_{\text {odd }}$, respectively. 
The condition $N=4,8,12, \ldots$ for the solution at $a=0$ to occur is a consequence of periodic boundary conditions. For all $N$ this leads to a total number of $N-2$ solutions. The two remaining ones are complex-conjugate solutions of eq. (5.8a).

TABLE I

\begin{tabular}{|c|c|c|}
\hline$N$ & $\operatorname{Re} a$ & $|\operatorname{Im} a|$ \\
\hline 6 & -0.86687 & +0.52026 \\
\hline 7 & -0.91792 & +0.52343 \\
\hline 8 & -0.93777 & +0.51256 \\
\hline 9 & -0.94284 & +0.50257 \\
\hline 10 & -0.94177 & +0.49684 \\
\hline 20 & -0.93750 & +0.49607 \\
\hline 30 & -0.93750 & +0.49608 \\
\hline 40 & -0.93750 & +0.49608 \\
\hline 50 & -0.93750 & +0.49608 \\
\hline$\infty$ & -0.93750 & +0.49608 \\
\hline
\end{tabular}

For finite $N$ these can easily be determined numerically. The results are given in table I for different values of $N$. In the limit of $N \rightarrow \infty$ the $B_{i}$ with $i \neq 0$ form a dense set on the $b$ axis between 1 and 3 . As a result, since $b=E-1$ this gives rise to a continuum of states with energies between $E=2$ and $E=4$. Moreover it is easily verified from the expressions for $c_{n}$ that the corresponding eigenstates are not normalizable and hence are scattering states of the system. The solution $B_{0}$ becomes isolated from the continuum and its position can easily be determined from (5.7a), which for $N \rightarrow \infty$ becomes

$$
a+2-\left[\frac{1}{2} a-\frac{1}{2}\left(1-1 / a^{2}\right)^{\frac{1}{2}}\right]=0,
$$

or $a=-\frac{17}{8}$. The coefficients are found immediately from eqs. (5.4) to be $c_{N-1}=0$, $c_{N}=-c_{N-2}=\Lambda\left(n_{1}\right)$. Because of periodic boundary conditions we have $\Lambda\left(n_{1}\right)$ $=\Lambda_{0} \mathrm{e}^{\mathrm{i} K n_{1}}$, where $K=2 \pi n / N, n=0,1,2, \ldots, N-1$. Furthermore, from (A.7) we have

$$
c_{n_{1}+\mu}\left(n_{1}\right)=\operatorname{sign} \mu\left[\left(a^{2}-1\right)^{\frac{1}{2}}-a\right]\left|a+\left(a^{2}-1\right)^{\frac{1}{2}}\right|^{|\mu|} A_{0} \mathrm{e}^{\mathrm{i} K n_{1}},
$$

with $\mu=n_{3}-n_{1}$. Note that $\left|a+\left(a^{2}-1\right)^{\frac{1}{2}}\right|=\frac{1}{4}$ for $a=-\frac{17}{8}$ so that we are dealing in this case with a bound-state type of solution. 
We now turn to the complex energy solutions. These energies are found from eq. (5.8a), which becomes in the limit $N \rightarrow \infty$

$$
a\left[\frac{1}{2} a+2+\frac{1}{2} a\left(1-1 / a^{2}\right)^{\frac{1}{2}}\right]+\frac{3}{2}=0,
$$

or

$$
1 / a=-\frac{5}{6} \pm \mathrm{i} \frac{1}{6} \sqrt{ } 7
$$

From (5.4) we have: $c_{N-2}=c_{N}=\Lambda_{0} \mathrm{e}^{i K n_{1}}, c_{N-1}=-(3 / a) \Lambda_{0} \mathrm{e}^{i K n_{1}}$ and from (A.7)

$$
c_{n_{1}+\mu}\left(n_{1}\right)=\left[a\left(1-1 / a^{2}\right)^{\frac{1}{2}}-a\right]^{-1}\left[a\left(1-1 / a^{2}\right)^{\frac{1}{2}}-a\right]^{|\mu|} \Lambda_{0} \mathrm{e}^{\mid K n_{1}} .
$$

For $a$ given by $(5.12 \mathrm{a}),\left|a\left(1-1 / a^{2}\right)^{\frac{3}{2}}-a\right|=\frac{1}{2}$, so that we are dealing with bound-state type of solutions, however at complex energies $E_{1} \approx 2.06+0.496 \mathrm{i}$ and $E_{2}=E_{1}^{*}$. It should be noted from table I, that the limit $N \rightarrow \infty$ is reached very fast.

The interpretation of the continuum $2 \leqslant E \leqslant 4$ may easily be given. For $n=2$ an unphysical bound state was found at $E=2$, in which two spin deviations occured at the same site. The energy spectrum $2 \leqslant E \leqslant 4$ corresponds to two spin deviations bound at one site, since only amplitudes $a_{n_{1} n_{1} n_{3}}$ can differ from zero, plus one freely moving spin deviation. The energy of such states is $E=E_{\mathrm{B}}^{(2)}$ $+E^{(1)}=2+(1-\cos k)$ with $k$ lying between $-\pi$ and $+\pi$, and this just produces a spectrum between $E=2$ and $E=4$.

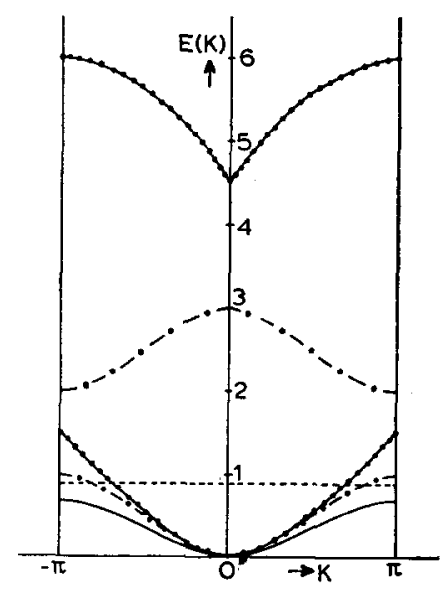

Fig. 3. The spectrum of $H_{\mathrm{D}}$ for real values of the energies as a function of total momentum $K$. Solid line is the position of the physical bound state, while the dashed line corresponds to the unphysical bound state. Furthermore, the dotted lines represent the upper and lower boundaries of the band of three free magnons, and similarly, the dash-dotted lines are the boundaries of one free magnon and a bound state of two magnons. 
Now we have found $N^{2}$ independent eigenstates of $H_{\mathrm{D}}$ and their energies in addition to the $\frac{1}{6} N(N-1)(N-2)$ states already found in the preceding section. Hence all eigenstates and eigenvalues of $H_{\mathrm{D}}$ in the subspace $n=3$ have been determined. In fig. 3 the spectrum of $H_{\mathrm{D}}$ in the subspace $n=3$ is shown, as far as the real cncrgy cigenvalues arc conccrned. The cncrgy is given as a function of total momentum $K$ of the three magnons.

(i) The continuum of physical scattering states of three magnons having the energy $\sum_{i}\left(1-\cos k_{i}\right)$ has as upper and lower boundary respectively:

$$
\begin{array}{ll}
3\left[1-\cos \frac{1}{3}(K-2 \pi)\right], & -\pi \leqslant K \leqslant 0, \\
3\left[1+\cos \frac{1}{3}(K-\pi)\right], & 0 \leqslant K \leqslant \pi ;
\end{array}
$$

and $3\left(1-\cos \frac{1}{3} K\right)$.

(ii) The continuum of physical states representing the scattering of a magnon on a bound state of two magnons having the cncrgy $1-\cos k_{1}+\frac{1}{2}\left(1-\cos K_{1}\right)$ has as upper and lower boundary respectively:

$$
\frac{3}{2}+\frac{\frac{5}{2}+2 \cos K}{(5+4 \cos K)^{\frac{1}{2}}} \quad \text { and } \quad \frac{3}{2}-\frac{\frac{5}{2}+2 \cos K}{(5+4 \cos K)^{\frac{1}{2}}}
$$

(iii) The continuum of unphysical scattering states with $2 \leqslant E \leqslant 4$ has been left out of the figure for reasons of clarity.

(iv) There is a physical bound state at energy $E_{p}=\frac{1}{3}(1-\cos K)$.

(v) There is an unphysical bound state at energy $E=0.875$. This bound state has the peculiar property of not depending on the total momentum $K$. It is amusing to note from the figure that for ccrtain valucs of $K$ the encrgy cigenvaluc of this state can be embedded in the continuum of scattering states, while it still constitutes a genuine bound state. In terms of the analytic properties of the $T$ matrix we have here the situation that it is possible to have a bound-state pole lying on a cut representing the scattering states.

(vi) Apart from the real energy values, there are two complex-conjugate energies $E_{1} \approx 2.06+0.496 \mathrm{i}$ and $E_{2}=E_{1}^{*}$. The corresponding eigenstates are normalizable. They arise from the fact that the Dyson hamiltonian is not hermitean.

6. Calculation of the three-body $T$ matrix near the bound-state energies. In the previous sections we have found two bound states of three magnons for the Dyson hamiltonian in one dimension, their energies being given by $E_{\mathrm{p}}=\frac{1}{3}(1-\cos K)$ and $E_{\mathrm{u}}=0.8750$ for the physical and unphysical one, respectively. Moreover we obtained two bound-state like solutions at complex energies $E_{\mathrm{c}}=2.06+0.496 \mathrm{i}$ and $E_{\mathrm{c}}^{*}=2.06-0.496 \mathrm{i}$. Since the wave functions have been calculated explicitly we are in a position to find the $T$ matrix analytically for energies near $E_{\mathrm{p}}, E_{\mathrm{u}}$ 
and $E_{\mathrm{c}}$. From the definition of the $T$ matrix it is clear that near a bound-state pole we have

$$
T(E) \equiv V+V G(E) V \approx V\left|\psi_{\mathrm{B}}\right\rangle \frac{1}{E-E_{\mathrm{B}}}\left\langle\psi_{\mathrm{B}}\right| V .
$$

Hence its momentum dependence can be expressed in terms of the bound-state wave function as

$$
\left\langle k_{1} k_{2} k_{3}|T(E)| \alpha\right\rangle \approx N\left\langle k_{1} k_{2} k_{3}|V| \psi_{\mathrm{B}}\right\rangle,
$$

where $N$ is a normalization factor. The result (6.1) holds also for the bound-state like solutions at $E_{\mathrm{c}}$ and $E_{\mathrm{c}}^{*}$, however the argument has to be treated more carefully. As demonstrated in appendix B the $T$ matrix has the following structure

$$
\left\langle k_{1} k_{2} k_{3}|T(E)| \alpha\right\rangle=\sum_{i=1}^{3} \cos p_{i} \Phi\left(K_{i}, E\right),
$$

where $\Phi\left(K_{1}, E\right)$ satisfies the following Faddeev equation:

$$
\begin{aligned}
\Phi\left(K_{1}, E\right)= & \frac{-2}{\pi D\left(t, K_{1}\right)} \int_{-\pi}^{+\pi} \mathrm{d} \tilde{K}_{2} \\
& \times \frac{\left[\cos \left(K-\tilde{K}_{2}-\frac{1}{2} K_{1}\right)-\cos \frac{1}{2} K_{1}\right] \cos \left(K_{1}+\frac{1}{2} \widetilde{K}_{2}-K\right)}{2 \cos \frac{1}{2} K_{1} \cos \left(K-\widetilde{K}_{2}-\frac{1}{2} K_{1}\right)-2 t} \\
& \times \Phi\left(\tilde{K}_{2}, E\right),
\end{aligned}
$$

with

$$
t=\frac{3}{2}-\frac{1}{2} \cos \left(K-K_{1}\right)-\frac{1}{2} E
$$

and

$$
D\left(t, K_{1}\right)=\frac{1}{\beta^{2}}\left(\frac{t\left[1-(\beta / t)^{2}\right]^{\frac{1}{2}}-t+\beta^{2}}{\left[1-(\beta / t)^{2}\right]^{\frac{1}{2}}}\right)
$$

with $\beta=\cos \frac{1}{2} K_{1}$.

The inhomogeneous term has been omitted in eq. (6.3) because we will be interested in this paper only for the bound-state solution of the Faddeev equations. This equation cannot be solved off-hand but by virtue of eq. (6.1) we may calculate $\Phi$ explicitly.

6.1. Calculation of $\Phi_{\mathrm{u}}\left(K_{1}, E\right)$. We recall from the previous section the bound-state condition for the unphysical state at $E_{\mathrm{u}}$ :

$$
\left(a^{2}-1\right)^{\frac{1}{2}}-a=4 \quad(a \equiv E-3)
$$


and its wave function:

$$
\begin{aligned}
a_{m_{1} m_{2} m_{3}}= & 0, \quad m_{1} \neq m_{2} \neq m_{3} \neq m_{1}, \\
a_{m_{1} m_{2} m_{2}}= & \operatorname{sign} \mu \Lambda_{\mathrm{v}}\left(1-\delta_{m_{1} m_{2}}\right)\left[\left(a^{2}-1\right)^{\frac{1}{2}}-a\right] \\
& \times \mid a+\left(a^{2}-1\right)^{\frac{1}{2}|| \mu \mid} \mathrm{e}^{i K m_{2}} \quad\left(\mu \equiv m_{1}-m_{2} ; a<0\right) .
\end{aligned}
$$

The expression for $V|\psi\rangle$, where $|\psi\rangle=\sum_{m_{1} m_{2} m_{3}} a_{m_{1} m_{2} m_{3}}\left|m_{1} m_{2} m_{3}\right\rangle$ is an arbitrary state (see appendix B):

$$
V|\psi\rangle=3 \sum_{\substack{m_{1} m_{2} \\ \text { all }}} \sum_{\Delta= \pm 1}\left(a_{m_{1} m_{2} m_{2}}-a_{m_{1} m_{2} m_{2}+\Delta}\right)\left|m_{1} m_{2} m_{2}+\Delta\right\rangle .
$$

As one sees,

$$
\begin{aligned}
\left\langle k_{1} k_{2} k_{3}|V| \psi\right\rangle= & 3 \sum_{\substack{m_{1} m_{2} \\
\mathbf{a} 11}} \sum_{\Delta} a_{m_{1} m_{2} m_{2}} \sum_{\mathbf{P}\left(p_{i}\right)} \mathrm{e}^{-i k_{p_{1}} m_{1}-\mathbf{i} K_{p_{1}} m_{2}-i k_{p_{3}} \Delta} \\
& -3 \sum_{m_{2}} \sum_{\Delta} a_{m_{2} m_{2} m_{2}+\Delta} \sum_{\mathbf{P}\left(p_{i}\right)} \mathrm{e}^{-\mathrm{i} K m_{2}-\mathrm{i} k_{p_{3}} \Delta} \\
& -3 \sum_{m_{2}} \sum_{\Delta} a_{m_{2} m_{2}+\Delta m_{2}+\Delta} \sum_{\mathbf{P}\left(p_{i}\right)} \mathrm{e}^{-1 K m_{2}-\mathbf{i} K_{p_{1}} A} .
\end{aligned}
$$

Into this expression all amplitudes of the bound-state wave function (6.6) are substituted. The calculation is now a straightforward matter. However, a few remarks are in order.

(i) The sums of permulations allow for a unique decomposition of $\langle\boldsymbol{k}|\boldsymbol{V}| \psi\rangle$ giving

$$
\langle\boldsymbol{k}|V| \psi\rangle=\delta\left(K-K^{\prime}\right) \sum_{i=1}^{3} \cos p_{i} f\left(K_{i}, E\right)
$$

by combining the permutations pairwise in an appropriate way. $f\left(K_{1}, E\right)$ may be identified as $\Phi_{\mathrm{u}}\left(K_{1}, E\right)$.

(ii) Since we take the limit $N \rightarrow \infty, \sum_{\text {all } m_{1} m_{2}}$ may be transformed into $\sum_{\text {all } m_{1}}$ $\sum_{\mathrm{all} \mu=m_{1} \cdots m_{2}}$, where $\sum_{m_{1}}$ provides $\delta\left(K-K^{\prime}\right)$ and $\sum_{\mu}$ gives rise to an infinite geometric series. The final result is

$$
f\left(K_{1}, E\right)=2 \mathrm{i} \Lambda_{0}\left(\left[\left(a^{2}-1\right)^{\frac{1}{2}}-a\right] \frac{\sin \left(K-K_{1}\right) \cos \frac{1}{2} K_{1}}{a+\cos \left(K-K_{1}\right)}+2 \sin \frac{1}{2} K_{1}\right),
$$

hence

$$
\Phi_{\mathrm{u}}\left(K_{1}, E\right)=F_{\mathrm{u}}\left(K_{1}, E\right) /\left[a+\cos \left(K-K_{1}\right)\right],
$$


with

$$
\begin{aligned}
F_{\mathrm{u}}\left(K_{1}, E\right)= & 2 \mathrm{i} \Lambda_{0}\left\{\left[\left(a^{2}-1\right)^{\frac{1}{2}}-a\right] \sin \left(K-K_{1}\right) \cos \frac{1}{2} K_{1}\right. \\
& \left.+2 \sin \frac{1}{2} K_{1}\left[a+\cos \left(K-K_{1}\right)\right]\right\},
\end{aligned}
$$

an analytic function of $K_{1}$, and the parameters $K$ and $E$.

6.2. Calculation of $\Phi_{\mathrm{c}}\left(K_{1}, E\right)$. From the preceding section we have for the bound-state like solutions at $E_{\mathrm{c}}$ and $E_{\mathrm{c}}^{*}$ the eigenvalue equation

$$
a\left[\frac{1}{2} a+2+\frac{1}{2} a\left(1-1 / a^{2}\right)^{\frac{1}{2}}\right]+\frac{3}{2}=0
$$

and the wave functions

$$
\begin{aligned}
& a_{n_{1}-1 n_{1} n_{1}}=a_{n_{1} n_{1} n_{1}+1}=\Lambda_{0} \mathrm{e}^{\mathrm{IKn} n_{1}}, \\
& a_{n_{1} n_{1} n_{1}}=-(3 / a) \Lambda_{0} \mathrm{e}^{\mathrm{I} K n_{1}}, \\
& a_{n_{1} n_{1} n_{3}}\left(n_{1}\right)=\left[a\left(1-1 / a^{2}\right)^{\frac{1}{2}}-a\right]^{-1}\left[a\left(1-1 / a^{2}\right)^{\frac{1}{2}}-a\right]^{|\mu|} \Lambda_{0} \mathrm{e}^{\mathrm{IKn} n_{1}}, \\
& |\mu|=\left|n_{3}-n_{1}\right| .
\end{aligned}
$$

$\left\langle k_{1} k_{2} k_{3}|V| \psi\right\rangle$ is exactly the same function of the amplitudes as in 6.1 , and remarks (i) and (ii) given there apply to its calculation. The final result is:

$$
\begin{aligned}
& 2 \Lambda_{0} \delta\left(K-K^{\prime}\right) \cos p_{1} \cos \frac{1}{2} K_{1} \\
& \quad \times\left[-\left(\frac{3}{a}+2\right)+\frac{a\left(1-1 / a^{2}\right)^{\frac{1}{2}}-a-\cos \left(K-K_{1}\right)}{\left[a\left(1-1 / a^{2}\right)^{\frac{3}{2}}-a\right]\left[a+\cos \left(K-K_{1}\right)\right]}\right],
\end{aligned}
$$

hence

$$
\Phi_{\mathrm{c}}\left(K_{1}, E\right)=F_{\mathrm{c}}\left(K_{1}, E\right) /\left[a+\cos \left(K-K_{1}\right)\right],
$$

with

$$
\begin{aligned}
F_{\mathrm{c}}\left(K_{1}, E\right)=2 & A_{0}\left[1-\frac{\cos \left(K-K_{1}\right)}{a\left(1-1 / a^{2}\right)^{\frac{1}{2}}-a}\right. \\
& \left.-\left(\frac{3}{a}+2\right)\left[a+\cos \left(K-K_{1}\right)\right]\right] \cos \frac{1}{2} K_{1},
\end{aligned}
$$

an analytic function of $K_{1}$, and the parameters $K$ and $E$.

6.3. Calculation of $\Phi_{\mathrm{p}}\left(K_{1}, E\right)$. Again our starting point is eq. (6.7). All the amplitudes that determine the physical bound state at $E_{\mathrm{p}}=\frac{1}{3}(1-\cos K)$ were 
calculated in section 4. Amplitudes of the Bethe type $a_{m_{1} m_{2} m_{3}}, m_{1} \neq m_{2} \neq m_{3} \neq m_{1}$ are given by $a_{m_{1} m_{2} m_{3}}=a_{m_{i_{1}} m_{t_{2} m_{i_{3}}}}^{\mathrm{B}}$; amplitudes of the type $a_{m_{1} m_{2} m_{2}}$, by $a_{m_{1} m_{2} m_{2}}$ $=a_{m_{1} m_{2} m_{2}}^{\mathrm{S}}+a_{m_{1} m_{2} m_{2}}^{\mathrm{E}}$. (For details about $a^{\mathbf{B}}, a^{\mathbf{S}}$ and $a^{\mathbf{E}}$ we refer to section 4.)

One now finds a large number of terms contributing to $\langle\boldsymbol{k}|V| \psi\rangle$ which are all of the same order of magnitude. All contributions are listed in appendix $C$ to give an idea of the amount of algebra involved. The details of the calculation, which is lengthy but straightforward, will be omitted. Remarks (i) and (ii) of section 6.1 apply here too and no additional troubles are encountered.

The final result reads:

$$
\begin{aligned}
\left\langle\boldsymbol{k}\left|T^{(1)}(E)\right| \psi_{\mathrm{p}}\right\rangle & =\delta\left(K-K^{\prime}\right) \cos p_{1} \Phi_{\mathrm{p}}\left(K_{1}, E\right)=\delta\left(K-K^{\prime}\right) \cos p_{1} \\
& \cdot \times \frac{F_{\mathrm{p}}\left(K_{1}, E\right)}{\left[\cos \left(K-K_{1}-u\right)-\cosh v\right]\left[a+\cos \left(K-K_{1}\right)\right]}
\end{aligned}
$$

$K$ is the total momentum, $u$ and $v$ are the parameters determining the bound state. $F_{\mathrm{p}}$ is an analytic function of $K_{1}$, and of the parameters $K, u, v$ and $E$. The expression for $F_{\mathrm{p}}$ is a very complicated combination of elementary functions and can be found in appendix $C$.

The structure of the functions $\Phi_{\mathrm{u}}, \Phi_{\mathrm{c}}$ and $\Phi_{\mathrm{p}}$ is quite striking. As one sees from (6.3) they give only rise to simple poles in the integrand of (6.3) at the solutions of $E-3+\cos \left(K-\tilde{K}_{2}\right)=0$ and $\cos \left(K-\tilde{K}_{2}-u\right)-\cosh v=0$ or $E-3$ $+\cos \left(K-\tilde{K}_{2}\right)=0$, respectively. The singularity at $\tilde{K}_{2}^{\mathrm{u}}$ determined by $a+\cos \left(K-\widetilde{K}_{2}^{\mathrm{u}}\right)=0$ is connected to the unphysical bound states; the one at $\widetilde{K}_{2}^{\mathrm{p}}$ determined by $\cos \left(K-\tilde{K}_{2}^{\mathrm{p}}-u\right)-\cosh v=0$ to the physical bound state. In $\Phi_{\mathrm{p}}$ the singularities related to both bound states at $K_{2}^{\mathrm{u}}$ and $\widetilde{K}_{2}^{\mathrm{p}}$ are present. This should be the case in principle for $\Phi_{\mathrm{u}}$ and $\Phi_{\mathrm{c}}$ too, but the singularity at $\tilde{K}_{2}^{\mathrm{p}}$ is absent, because all $a_{m_{1} m_{2} m_{3}}\left(m_{1} \neq m_{2} \neq m_{3} \neq m_{1}\right)$ are zero.

7. Solution of the Faddeev equation. In this section we shall show by explicit integration, that for $E=E_{\mathrm{u}} \Phi_{\mathrm{u}}$ given by (6.8) is an eigenfunction of the integral equation (6.3). After substitution of (6.8) into (6.3) we have to verify that $F_{u}$ defined by eq. (6.9) is a solution of

$$
\begin{aligned}
\frac{F_{\mathrm{u}}\left(K_{1}, E\right)}{a+\cos \left(K-K_{1}\right)}= & \frac{1}{\pi D\left(t, K_{1}\right)} \int_{-\pi}^{+\pi} \mathrm{d} \tilde{K}_{2} \\
& \times \frac{\left[\cos \left(K-\widetilde{K}_{2}-\frac{1}{2} K_{1}\right)-\cos \frac{1}{2} K_{1}\right] \cos \left(K_{1}-K+\frac{1}{2} \widetilde{K}_{2}\right)}{t-\cos \frac{1}{2} K_{1} \cos \left(K-\widetilde{K}_{2}-\frac{1}{2} K_{1}\right)} \\
& \times \frac{F_{\mathrm{u}}\left(\tilde{K}_{2}, E\right)}{a+\cos \left(K-\tilde{K}_{2}\right)} .
\end{aligned}
$$


Since $F_{\mathrm{u}}$ has the important property of being analytic in $\widetilde{K}_{2}$, the integral can be carried out explicitly. Introducing the variable $z=\mathrm{e}^{1 \tilde{K}_{2}}$, the integral in (7.1) may be transformed into

$$
\oint \frac{\mathrm{d} z}{z^{2}} \frac{P^{(6)}(z)}{Q^{(2)}(z) R^{(2)}(z)},
$$

where the integration path is along the unit circle in the complex $\widetilde{K}_{2}$ plane. $P^{(6)}$ is a sixth-order polynomial in $z$ corresponding to the numerator of (7.1), while $Q^{(2)}$ and $R^{(2)}$ are second-order polynomials in $z$ given by

$$
Q^{(2)}(z)=\frac{1}{2} \cos \frac{1}{2} K_{1} \mathrm{e}^{1\left(K-\frac{1}{2} K_{1}\right)}-t z+\frac{1}{2} \cos \frac{1}{2} K_{1} \mathrm{e}^{-\mathrm{i}\left(K-\frac{1}{2} K_{1}\right)} z^{2}
$$

and

$$
R^{(2)}(z)=\frac{1}{2} \mathrm{e}^{\mathrm{i} K}+a z+\frac{1}{2} \mathrm{e}^{-i K} z^{2} .
$$

Applying Cauchy's theorem to (7.2) gives for the right-hand side of (7.1):

$$
\begin{aligned}
& \frac{2 \mathrm{i} \Lambda_{0}}{D\left(t, K_{1}\right)} \sin \frac{1}{2} K_{1}\left\{\left[\left(a^{2}-1\right)^{\frac{1}{2}}-a\right]\left[\beta^{2}\left(t^{2}-\beta^{2}\right)^{\frac{1}{2}}\right]^{-1}\right. \\
& \quad \times\left[t\left(\beta^{2}-t\right)-2\left(a^{2}-1\right)^{\frac{1}{2}} \beta^{2}\left(t^{2}-\beta^{2}\right)^{\frac{1}{2}}\right. \\
& \left.\quad+t\left(t^{2}-\beta^{2}\right)^{\frac{1}{2}}-\beta^{2}\left(t^{2}-\beta^{2}\right)^{\frac{1}{2}}-2 a \beta^{2}\left(t^{2}-\beta^{2}\right)^{\frac{1}{2}}\right] \\
& \left.\quad+\left[\beta^{2}\left(t^{2}-\beta^{2}\right)^{\frac{1}{2}}\right]^{-1}\left[2 t\left(t-\beta^{2}\right)+2 \beta^{2}\left(t^{2}-\beta^{2}\right)^{\frac{1}{2}}-2 t\left(t^{2}-\beta^{2}\right)^{\frac{1}{1}}\right]\right\} \\
& \quad+\frac{2 \mathrm{i} \Lambda_{0}}{D\left(t, K_{1}\right)} \sin \left(K-K_{1}\right)\left(\frac{2\left(t-\beta^{2}\right)-2\left(t^{2}-\beta^{2}\right)^{\frac{1}{2}}}{\beta\left(t^{2}-\beta^{2}\right)^{\frac{1}{2}}}\right),
\end{aligned}
$$

where $\beta \equiv \cos \frac{1}{2} K_{1}$. Substituting (6.4b) for $D\left(t, K_{1}\right)$ one finds for the term with $\sin \left(K-K_{1}\right) \quad 8 \mathrm{i} \Lambda_{0} \cos \frac{1}{2} K_{1} \sin \left(K-K_{1}\right) /\left[a+\cos \left(K-K_{1}\right)\right]$. Comparing this expression with the term of the left-hand side of (7.1) with the same $K_{1}$ dependence, we find that they are the same, if

$$
\left(a^{2}-1\right)^{\frac{1}{2}}-a=4,
$$

which is precisely the bound-state condition. Similarly the terms of eq. (7.1) with $\sin \frac{1}{2} K_{1}$ as a factor agree if (7.6) holds.

For $F_{\mathrm{c}}\left(K_{1}, E\right)$ the residues of the integral in (7.1) at the same poles as in the preceding case have to be calculated. One finds that (7.1) holds with (6.13) for $F_{\mathrm{c}}$ precisely at the solutions of (6.10). 
For the physical bound state the same calculation could be carried through, since the resulting integrand for this case is also analytic in the variable $z=\mathrm{e}^{\mathrm{i} \tilde{\kappa}_{2}}$ except for a finite number of poles. Because of the vast amount of algebra, we have not done this. Instead, we convinced ourselves by numerical integration, that $\Phi_{\mathrm{p}}\left(K_{1}, E\right)$ found in the previous section is indeed a solution of the Faddeev equation at the bound state energy $E_{\mathrm{p}}=\frac{1}{3}(1-\cos K)$. For completeness it was also checked numerically that $\Phi_{\mathrm{u}}$ and $\Phi_{\mathrm{c}}$ are solutions of (6.3). In order to get some quantitative idea how the solution $F_{\mathrm{p}}$ behaves, it has been plotted for various values of $K$ in fig. 4.

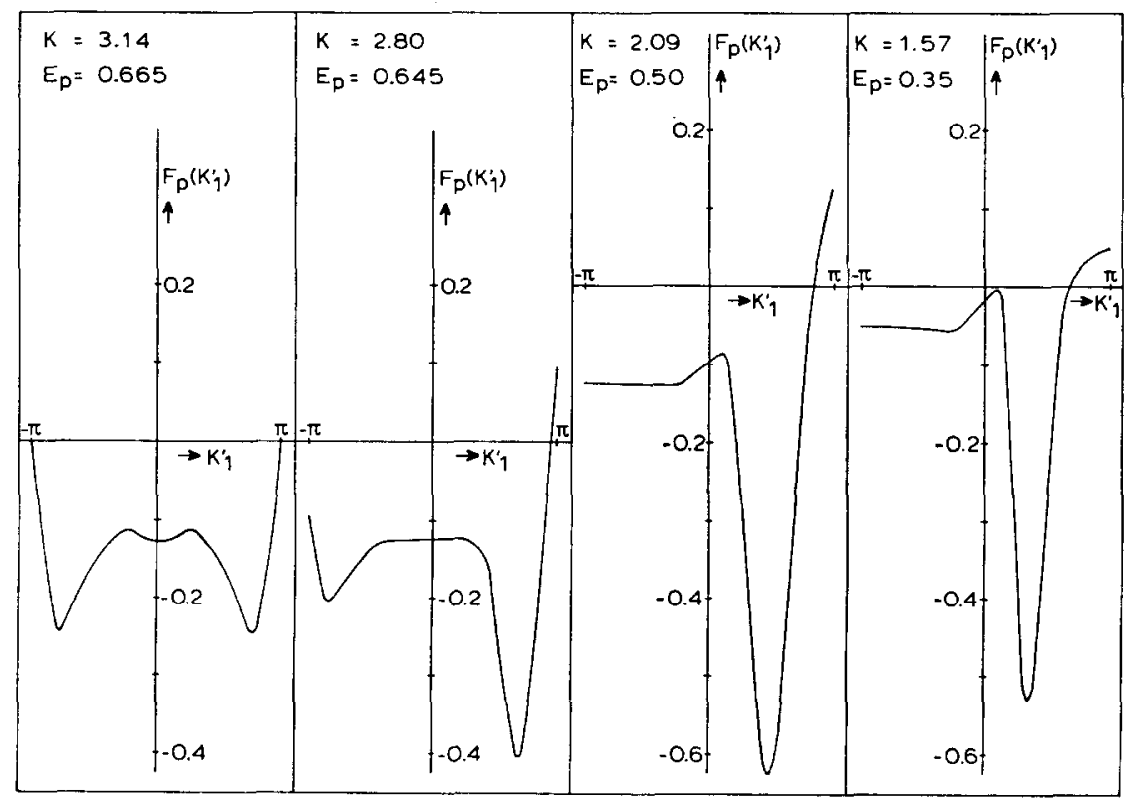

Fig. 4. The function $F_{\mathrm{p}}$ for various values of total momentum $K$.

8. Concluding remarks. In this paper we have determined the eigenvalues and eigenstates of two and three magnons in a one-dimensional system described by the Dyson hamiltonian. The bound-state wave functions are used to construct the eigenfunctions of the Faddeev equation in the bound-state region. These solutions have a very simple mathematical structure, namely they are analytic in the variable $z=\mathrm{e}^{\tilde{T}}$ except for a finite number of poles. Similar results have been found by Dodd for a one-dimensional system of three particles interacting through delta-function potentials. Majumdar, who wrote down the explicit form of the Faddeev equation for the Dyson hamiltonian verified numerically the existence of a bound state at the same energy as Bethe's solution in the case of the one- 
dimensional Heisenberg chain, but overlooked the existence of additional bound states.

The question in how far we may use the Faddeev equations for the Dyson hamiltonian as a starting point for finding possible three-magnon bound states in higher dimension, for the Heisenberg system is intimately related to the problem of distinguishing between physical and nonphysical solutions. Obviously, we may beforehand exclude the complex-energy states as physical since they are a consequence of the nonhermiticity of the hamilionian. With respect to the real energy solutions in principle this can be done by showing that the determined state has no components in the physical Hilbert space. It is worth mentioning that in the one-dimensional model we have studied here, the energies of the unphysical bound states are independent of the total momentum. This feature might hold also in higher dimension, which would then be a simple way of distinguishing between the physical and unphysical states.

TABLE II

\begin{tabular}{|c|c|c|}
\hline \multicolumn{3}{|c|}{$\begin{array}{l}\text { Position of the energy } E_{\mathrm{p}} \text { at } K=\pi \text { of the } \\
\text { physical bound state and the energy } E_{\mathrm{u}} \text { of the } \\
\text { unphysical bound state at real energy as a } \\
\text { function of anisotropy } \sigma \text {. }\end{array}$} \\
\hline$\sigma$ & $E_{\mathrm{p}}$ & $E_{\mathrm{u}}$ \\
\hline 1.0 & 0.667 & 0.875 \\
\hline 0.8 & 0.773 & 0.923 \\
\hline 0.6 & 0.862 & 0.955 \\
\hline 0.4 & 0.934 & 0.980 \\
\hline 0.2 & 0.983 & 0.994 \\
\hline 0.0 & 1.00 & 1.00 \\
\hline
\end{tabular}

Finally the Faddeev formalism was used recently ${ }^{7}$ ) to study the case where the exchange is anisotropic $J_{x}=J_{y}=\sigma J, J_{z}=J$. We have also studied this case and have found in addition to the physical bound state also an unphysical solution at real energy. For $K=\pi$ the results are given in table II. Also in this case it was found that for a fixed $\sigma$ the energy of the unphysical bound state is independent of $K$. It should be noted that also in this case the analytic solution can be constructed in the same way as described in this paper for the isotropic one, starting from the solutions known for the anisotropic one-dimensional Heisenberg chain $^{8,9}$ ). 


\section{APPENDIX A}

In this appendix we shall be concerned with the solution of the set of linear equations (4.15). In matrix notation we have for $E e=\varepsilon$ :

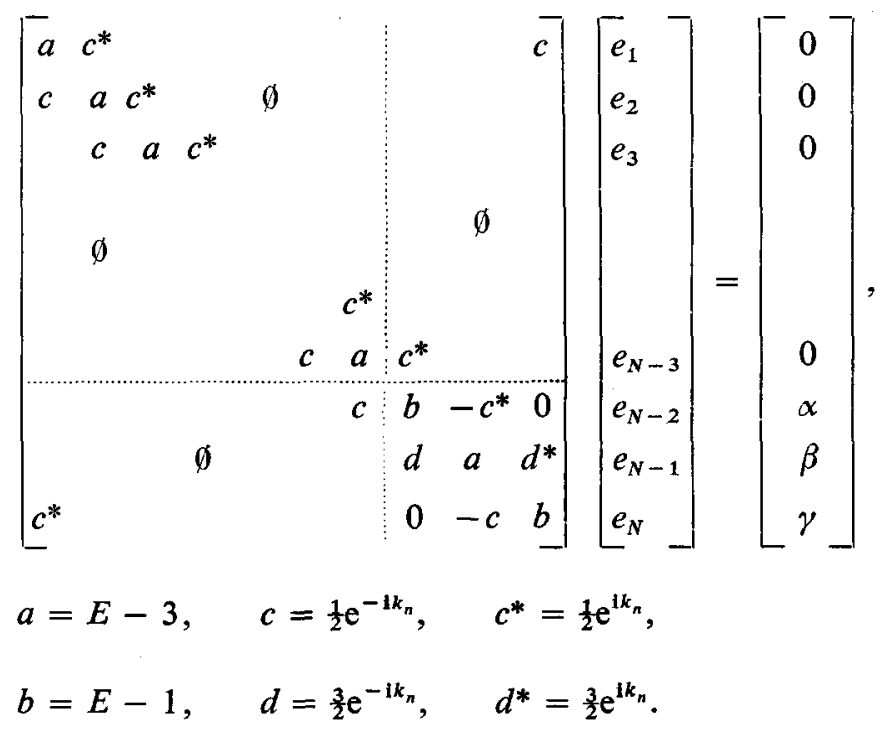

First we consider the eigenvalue problem of the $(N-3) \times(N-3)$ matrix $E_{0} \varphi=\lambda \varphi$, or

$$
\left[\begin{array}{llllll}
a & c^{*} & & & & \\
c & a & c^{*} & & \emptyset & \\
& c & a & c^{*} & & \\
& & & & & \\
& \emptyset & & & & \\
& & & & & c^{*} \\
- & & & & & a
\end{array}\right]\left[\begin{array}{l}
\varphi_{1} \\
\varphi_{2} \\
\varphi_{3} \\
\varphi_{N-3}
\end{array}\right]=\lambda\left[\begin{array}{l}
\varphi_{3} \\
\varphi_{2} \\
\varphi_{N-3}
\end{array}\right] .
$$

Solution of the equations $\frac{1}{2} \mathrm{e}^{-i k_{n}} \varphi_{m-1}+a \varphi_{m}+\frac{1}{2} \mathrm{e}^{i k_{n}} \varphi_{m+1}=\lambda \varphi_{m}$ yields $\varphi_{m}$ $=\mathrm{e}^{-1 k_{n} m}\left(A \mathrm{e}^{\mathrm{i} m y}+B \mathrm{e}^{-i m y}\right)$ where $\cos y=\lambda-a$. From the boundary conditions $\varphi_{0}=0$ and $\varphi_{N-2}=0$, one finds respectively: $B=-A ; y_{v}=\pi \nu /(N-2)$ where $\boldsymbol{\nu}=1,2, \ldots, N-3$ are the possible values of $y$; or $\lambda_{v}=a+\cos [\pi v /(N-2)]$ where $\nu=1,2, \ldots, N-3$.

From the normalization condition for the eigenvectors $\sum_{p=1}^{N-3} \varphi_{p}^{\nu *} \varphi_{p}^{\nu}=1$ we find $A=[2 /(N-2)]^{\frac{1}{2}}$. 
So one obtains the following orthonormal eigenvectors $\left\{\varphi^{\nu}\right\}$ :

$$
\begin{aligned}
& \left(\varphi_{1}^{v}, \ldots, \varphi_{p}^{v}, \ldots\right) \\
& \quad=[2 /(N-2)]^{\frac{1}{2}}\left(\mathrm{e}^{-\mathrm{i} k_{n}} \sin y_{v}, \ldots, \mathrm{e}^{-1 k_{n} p} \sin p y_{v}, \ldots\right), \\
& v=1,2, \ldots, N-3, \quad p=1,2, \ldots, N-3,
\end{aligned}
$$

at the eigenvalues $\lambda_{v}=a+\cos y_{v}$,

$$
v=1,2, \ldots, N-3, \quad \text { with } \quad y_{v}=\pi v /(N-2) .
$$

Next we perform a unitary transformation on $E$ defined by:

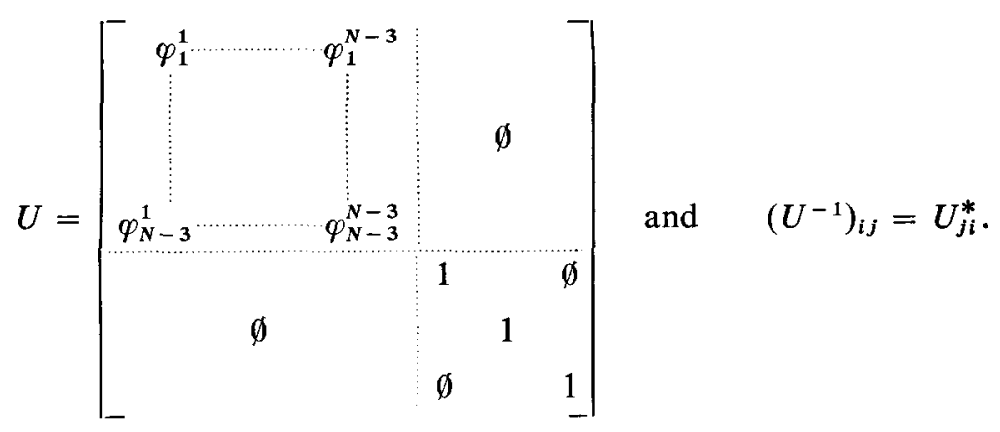

We get:

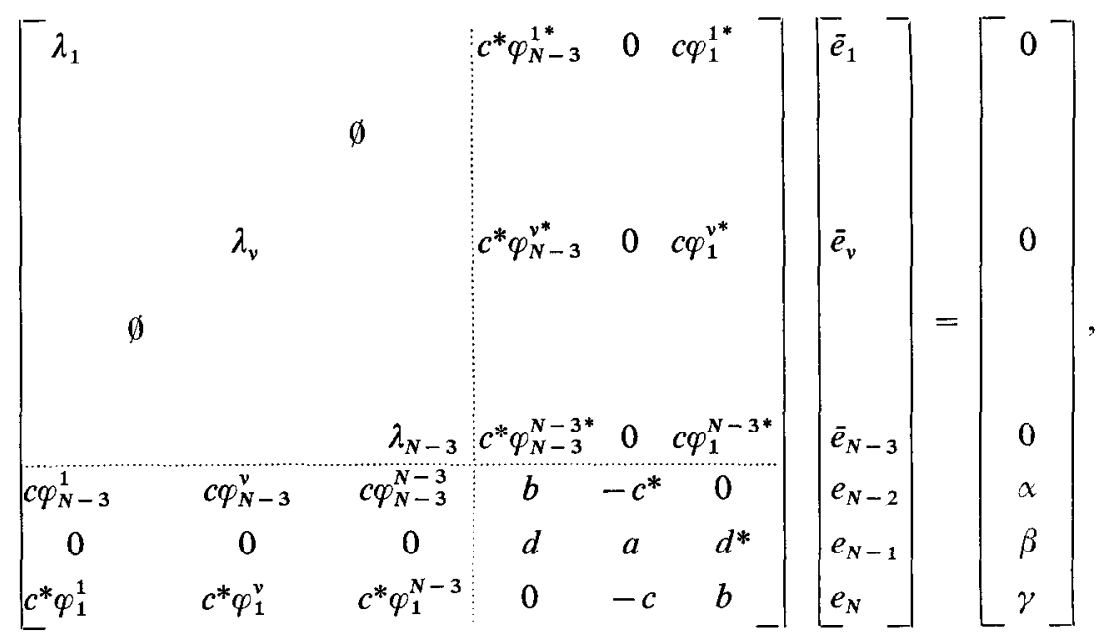


with $\bar{e}_{v}=\sum_{i=1}^{N-3} \varphi_{i}^{v *} e_{i}$ or explicitly:

$$
\begin{aligned}
& \lambda_{\nu} \bar{e}_{\nu}+c^{*} \varphi_{N-3}^{\nu^{*}} e_{N-2}+c \varphi_{1}^{\nu^{*}} e_{N}=0, \\
& c \sum_{\mu=1}^{N-3} \varphi_{N-3}^{\mu} \bar{e}_{\mu}+b e_{N-2}-c^{*} e_{N-1}=\alpha, \\
& d e_{N-2}+a e_{N-1}+d^{*} e_{N}=\beta, \\
& c^{*} \sum_{\mu=1}^{N-3} \varphi_{1}^{\mu} \bar{e}_{\mu}-c e_{N-1}+b e_{N}=\gamma .
\end{aligned}
$$

Eliminating $\bar{e}_{\mu}$ from eqs. (A.4b), (A.4d) with the aid of eq. (A.4a) gives for (A.4b)(A.4d):

$$
\begin{aligned}
& (b-\Gamma) e_{N-2}-c^{*} e_{N-1}-\Delta e_{N}=\alpha, \\
& d e_{N-2}+a e_{N-1}+d^{*} e_{N}=\beta, \\
& -\Delta^{*} e_{N-2}-c e_{N-1}+(b-\Gamma) e_{N}=\gamma,
\end{aligned}
$$

with

$$
\Gamma=|c|^{2} \sum_{v=1}^{N-3}\left|\varphi_{1}^{v}\right|^{2} / \lambda_{v} \quad \text { and } \quad \Delta=c^{2} \sum_{v=1}^{N-3} \varphi_{1}^{v^{*}} \varphi_{N-3}^{v} / \lambda_{v}
$$

Having solved $e_{N-2}, e_{N-1}$ and $e_{N}$ from (A.5a)-(A.5c), all $e_{i}(i<N-3)$ may be solved from the $N-3$ equations (A.4a). Let us suppose that $N$ is even. From (A.4a) we obtain:

$$
\begin{aligned}
e_{k}= & -\frac{1}{2} \mathrm{e}^{i k_{n}(N-3)-i k k_{n}}\left(\sum_{v=1}^{N-3} \varphi_{N-3}^{v} \varphi_{k}^{v} / \lambda_{v}\right) e_{N-2} \\
& -\frac{1}{2} \mathrm{e}^{-\mathrm{i}(k-1) k_{n}}\left(\sum_{v=1}^{N-3} \varphi_{1}^{v} \varphi_{k}^{v} / \lambda_{v}\right) e_{N} .
\end{aligned}
$$

Then all $e_{i}(i \neq N-2, N-1, N)$ are expressed in terms of $e_{N-2}, e_{N-1}$ and $e_{N}$ by

$$
\begin{aligned}
e_{k}= & -\frac{\mathrm{e}^{\mathrm{i} k_{n}(N-2-k)}}{N-2} \sum_{\nu=1}^{N-3}(-1)^{\nu+1} \lambda_{v}^{-1} \sin \frac{\pi \nu}{N-2} \sin \frac{k \pi \nu}{N-2} e_{N-2} \\
& -\frac{\mathrm{e}^{-1 k_{n} k}}{N-2} \sum_{\nu=1}^{N-3} \lambda_{\nu}^{-1} \sin \frac{\pi \nu}{N-2} \sin \frac{k \pi \nu}{N-2} e_{N},
\end{aligned}
$$




$$
\begin{aligned}
& e_{N-k-2}=-\frac{\mathrm{e}^{\mathrm{i} k k_{n} k}}{N-2} \sum_{\nu=1}^{N-3} \lambda_{v}^{-1}(-1)^{2(v+1)} \sin \frac{\pi \nu}{N-2} \sin \frac{k \pi \nu}{N-2} e_{N-2} \\
&-\frac{\mathrm{e}^{-\mathrm{i}(N-2-k) k_{n}}}{N-2} \sum_{v=1}^{N-3} \lambda_{v}^{-1}(-1)^{v+1} \sin \frac{k \pi \nu}{N-2} \sin \frac{\pi \nu}{N-2} e_{N}, \\
& k<\frac{1}{2}(N-1) .
\end{aligned}
$$

Taking $N \rightarrow \infty$, sums over $v$ in which the terms have alternating sign vanish, and

$$
\begin{aligned}
& -\frac{1}{N-2} \sum_{v=1}^{N-3} \lambda_{v}^{-1} \sin \frac{\pi v}{N-2} \sin \frac{k \pi v}{N-2} \\
& \underset{N \rightarrow \infty}{\longrightarrow}-\frac{1}{\pi} \int_{0}^{\pi} \frac{\sin x \sin k x}{a+\cos x} \mathrm{~d} x \\
& \quad=-\left[a\left(1-1 / a^{2}\right)^{\frac{1}{2}}+a\right]\left[a\left(1-1 / a^{2}\right)^{\frac{1}{2}}-a\right]^{k+1} .
\end{aligned}
$$

Hence, renumbering the two spin deviations at site $N-1$ back to the point $n_{1}$ in the chain, we obtain:

$$
\begin{aligned}
& e_{n_{1}+\mu}=-\left[a\left(1-1 / a^{2}\right)^{\frac{1}{2}}+a\right]\left[a\left(1-1 / a^{2}\right)^{\frac{1}{2}}-a\right]^{\mu} \mathrm{e}^{-i k_{n}(\mu-1)} e_{n_{1}+1}, \\
& e_{n_{1}-\mu}=-\left[a\left(1-1 / a^{2}\right)^{\frac{1}{2}}+a\right]\left[a\left(1-1 / a^{2}\right)^{\frac{1}{2}}-a\right]^{\mu} \mathrm{e}^{i k_{n}(\mu-1)} e_{n_{1}-1},
\end{aligned}
$$

where $\mu=k+1>0$ is the distance of the third spin deviation to $n_{1}$. (A.7) holds too for $\mu=1$.

Eqs. (A.5) take on a particularly simple form in the limit $N \rightarrow \infty$ since:

$$
\begin{aligned}
& \Delta= \frac{\mathrm{e}^{-i(N-2) k_{n}}}{2(N-2)} \sum_{v=1}^{N-3}\left(\lambda_{v}\right)^{-1}(-1)^{v+1} \sin ^{2} \frac{\pi v}{N-2} \underset{N \rightarrow \infty}{\longrightarrow} 0, \\
& \Gamma=\frac{1}{2(N-2)} \sum_{v=1}^{N-3} \sin ^{2}[\pi v /(N-2)]\{a+\cos [\pi v /(N-2)]\}^{-1} \\
& \rightarrow \frac{1}{2 \pi} \int_{0}^{\pi} \frac{\sin ^{2} x}{a+\cos x} \mathrm{~d} x=\frac{a}{2}-\frac{a}{2}\left(1-\frac{1}{a^{2}}\right)^{\frac{1}{2}} .
\end{aligned}
$$

Now because $\Delta=0$ eqs. (A.5) become:

$$
\begin{aligned}
& (b-\Gamma) e_{N-2}-\frac{1}{2} \mathrm{e}^{1 k_{n}} e_{N-1}=\alpha, \\
& \frac{3}{2} \mathrm{e}^{-1 k_{n}} e_{N-2}+a e_{N-1}+\frac{3}{2} \mathrm{e}^{1 k_{n}} e_{N}=\beta, \\
& -\frac{1}{2} \mathrm{e}^{-1 k_{n}} e_{N-1}+(b-\Gamma) e_{N}=\gamma .
\end{aligned}
$$




\section{APPENDIX B}

Derivation of the Faddeev equation. To establish our notation we give in this appendix a simple derivation of the integral equation satisfied by the three-particle $T$ matrix in the one-dimensional case. For an arbitrary state $|\psi\rangle=\sum_{m_{1} m_{1} m_{3}} a_{m_{1} m_{2} m_{3}}$ $\times\left|m_{1} m_{2} m_{3}\right\rangle H_{\mathrm{D}}|\psi\rangle$ can be written as $H_{\mathbf{D}}|\psi\rangle=H_{0}|\psi\rangle+V|\psi\rangle$ with

$$
\begin{aligned}
H_{0}|\psi\rangle= & \sum_{m_{1} m_{2} m_{3}}\left(3 J a_{m_{1} m_{2} m_{3}}\left|m_{1} m_{2} m_{3}\right\rangle\right. \\
& \left.-\frac{1}{2} J \sum_{\mathbf{C P}(i j k) \Delta} \sum_{\Delta} a_{m_{i} m_{j} m_{k}}\left|m_{i}+\Delta m_{j} m_{k}\right\rangle\right),
\end{aligned}
$$

where $\sum_{\mathrm{CP}(i J k)}$ is a sum over cyclic permutation of 1,2 and 3 , and

$$
V|\psi\rangle=3 J \sum_{m_{1} m_{2}} \sum_{\Delta}\left(a_{m_{1} m_{2} m_{2}}-a_{m_{1} m_{2} m_{2}+\Delta}\right)\left|m_{2} m_{2}+\Delta m_{1}\right\rangle .
$$

(B.1a) and (B.1b) follow from straightforward calculation of $H_{\mathrm{D}}|\psi\rangle$. The term $H_{0}$ has been chosen such that it is diagonal in the momentum representation. New momenta are defined in terms of the momenta $k_{1}, k_{2}$ and $k_{3}$ of the individual magnons as follows: $K_{1}=k_{2}+k_{3}, p_{1}=\frac{1}{2}\left(k_{2}-k_{3}\right), K_{2}=k_{1}+k_{3}, p_{2}=\frac{1}{2}$ $\times\left(k_{3}-k_{1}\right), K_{3}=k_{1}+k_{2}, p_{3}=\frac{1}{2}\left(k_{1}-k_{2}\right)$. The matrix elements of the Dyson potential between three-magnon states read as follows:

$$
\begin{aligned}
\left\langle k_{1}^{\prime} k_{2}^{\prime} k_{3}^{\prime}|V| k_{1} k_{2} k_{3}\right\rangle= & -(4 J / N) \delta\left(K-K^{\prime}\right) \\
& \times \sum_{i, j=1}^{3}\left(\cos p_{i} \cos p_{j}^{\prime}-\cos \frac{1}{2} K_{i} \cos p_{j}^{\prime}\right) \delta\left(K_{i}-K_{j}^{\prime}\right) .
\end{aligned}
$$

These are obtained easily by Fourier transformation of $\boldsymbol{V}$ in the site representation:

$$
\left|r_{1} r_{2} r_{3}\right\rangle=\left(1 / N^{3 / 2}\right) \sum_{k_{1} k_{2} k_{3}} \exp \left(-\mathrm{i} \sum_{n=1}^{3} k_{n} r_{n}\right)\left|k_{1} k_{2} k_{3}\right\rangle .
$$

One sees from (B.2) that $\left\langle\boldsymbol{k}^{\prime}|V| \boldsymbol{k}\right\rangle$ may be written as:

$$
\left\langle\boldsymbol{k}^{\prime}|V| \boldsymbol{k}\right\rangle=\sum_{m=1}^{3}{ }_{m}\left\langle K K_{m}^{\prime} p_{m}^{\prime}\left|V_{m}\right| \boldsymbol{k}\right\rangle,
$$

where

$$
\begin{aligned}
& { }_{m}\left\langle K K_{m}^{\prime} p_{m}^{\prime}\left|V_{m}\right| \boldsymbol{k}\right\rangle \\
& \quad=-(4 J / N) \cos p_{m}^{\prime}\left(\sum_{i}\left(\cos p_{t}-\cos \frac{1}{2} K_{i}\right) \delta\left(K_{m}^{\prime}-K_{t}\right)\right)
\end{aligned}
$$

for $m=1,2,3$, where we put $K=K^{\prime}$. The states $\left|K K_{m}^{\prime} p_{m}^{\prime}\right\rangle_{m}$ are the well-known states in which particle $m$ plays a special role. 
Analogously to (B.4) the two-magnon $T$ matrix in the three-magnon subspace may be split into three parts:

$$
\left\langle\boldsymbol{k}^{\prime}|T| \boldsymbol{k}\right\rangle=\sum_{m=1}^{3}{ }_{m}\left\langle K K_{m}^{\prime} p_{m}^{\prime}\left|T_{m}\right| \boldsymbol{k}\right\rangle,
$$

each of which obeys the following Lippmann-Schwinger equation:

$$
\begin{aligned}
{ }_{m}\left\langle K K_{m}^{\prime} p_{m}^{\prime}\left|T_{m}\right| \boldsymbol{k}\right\rangle= & { }_{m}\left\langle K K_{m}^{\prime} p_{m}^{\prime}\left|V_{m}\right| \boldsymbol{k}\right\rangle \\
& +\frac{1}{2} \sum_{\tilde{K}_{m}^{\prime} \tilde{p}_{m}}{ }_{m}\left\langle K K_{m}^{\prime} p_{m}^{\prime}\left|V_{m}\right| K \tilde{K}_{m} \tilde{p}_{m}\right\rangle_{m} \frac{{ }_{m}\left\langle K \tilde{K}_{m} \tilde{p}_{m}\left|T_{m}\right| \boldsymbol{k}\right\rangle}{E-E\left(K, \tilde{K}_{m}, \tilde{p}_{m}\right)}
\end{aligned}
$$

Substitution of (B.5) into (B.7) shows that the solution has the following general form:

$$
{ }_{m}\left\langle K K_{m}^{\prime} p_{m}^{\prime}\left|T_{m}\right| \boldsymbol{k}\right\rangle=\cos p_{m}^{\prime} \Phi\left(K_{m}^{\prime}, E\right),
$$

where $\Phi\left(K_{m}^{\prime}, E\right)$ obeys the following equation:

$$
\begin{aligned}
\Phi\left(K_{m}^{\prime}, E\right)= & -(4 J / N) \sum_{i}\left(\cos p_{i}-\cos \frac{1}{2} K_{i}\right) \delta\left(K_{m}^{\prime}-K_{i}\right) \\
& -\frac{2 J}{N} \sum_{\widetilde{K}_{m} \tilde{p}_{m}}\left(\cos \tilde{p}_{m}-\cos \frac{1}{2} \widetilde{K}_{m}\right) \delta\left(\widetilde{K}_{m}-K_{m}^{\prime}\right) \frac{\cos \tilde{p}_{m} \Phi\left(\widetilde{K}_{m}, E\right)}{E-E\left(K, \widetilde{K}_{m}, \tilde{p}_{m}\right)} .
\end{aligned}
$$

Performing $\sum \tilde{\kappa}_{m}$ one obtains immediately:

$$
\begin{aligned}
\Phi\left(K_{m}^{\prime}, E\right)= & \left(-(4 J \mid N) \sum_{i}\left(\cos p_{i}-\cos \frac{1}{2} K_{i}\right) \delta\left(K_{m}^{\prime}-K_{i}\right)\right) \\
& \times\left(1+\frac{2 J}{N} \sum_{\tilde{p}_{m}} \frac{\left(\cos \tilde{p}_{m}-\cos \frac{1}{2} K_{m}^{\prime}\right) \cos \tilde{p}_{m}}{E-E\left(K, K_{m}^{\prime}, \tilde{p}_{m}\right)}\right)^{-1} .
\end{aligned}
$$

Hence for $m=1,2,3$

$$
{ }_{m}\left\langle K K_{m}^{\prime} p_{m}^{\prime}\left|T_{m}\right| \boldsymbol{k}\right\rangle={ }_{m}\left\langle K K_{m}^{\prime} p_{m}^{\prime}\left|V_{m}\right| \boldsymbol{k}\right\rangle \mid D\left(E, K_{m}^{\prime}\right),
$$

where $D$ is the denominator of (B.9).

Similarly the three-magnon $T$ matrix may be calculated from Faddeev's equation (we concentrate on $T^{(1)}$ ):

$$
\begin{aligned}
{ }_{1}\left\langle K K_{1}^{\prime} p_{1}^{\prime}\left|T^{(1)}\right| k\right\rangle= & { }_{1}\left\langle K K_{1}^{\prime} p_{1}^{\prime}\left|T_{1}\right| k\right\rangle \\
& +\frac{1}{2} \sum_{i=2}^{3} \sum_{\tilde{K}_{l} \tilde{p}_{i}}{ }_{1}\left\langle K K_{1}^{\prime} p_{1}^{\prime}\left|T_{1}\right| K \widetilde{K}_{i} \tilde{p}_{i}\right\rangle_{i} \frac{\left\langle K \tilde{K}_{i} \tilde{p}_{i}\left|T^{(i)}\right| k\right\rangle}{E-E\left(\tilde{K}_{i}, \tilde{p}_{i}\right)}
\end{aligned}
$$


The complete $T$ matrix is $T=\sum_{m=1}^{3} T^{(m)}$, and equations similar to (B.11) hold for $T^{(2)}$ and $T^{(3)}$.

Since $\left|K \widetilde{K}_{2} \tilde{p}_{2}\right\rangle_{2}=\left|K \widetilde{K}_{1}^{(2)} \tilde{p}_{1}^{(2)}\right\rangle_{1}$ with

$$
\tilde{K}_{1}^{(2)}=K-\frac{1}{2} \tilde{K}_{2}+\tilde{p}_{2} \quad \text { and } \quad \tilde{p}_{1}^{(2)}=\frac{1}{2} K-\frac{3}{4} \widetilde{K}_{2}-\frac{1}{2} \tilde{p}_{2},
$$

and $\left|K \widetilde{K}_{3} \tilde{p}_{3}\right\rangle_{3}=\left|K \widetilde{K}_{1}^{(3)} \tilde{p}_{1}^{(3)}\right\rangle_{1}$ with

$$
\tilde{K}_{1}^{(3)}=K-\frac{1}{2} \widetilde{K}_{3}-\tilde{p}_{3} \quad \text { and } \quad \tilde{p}_{1}^{(3)}=-\frac{1}{2} K+\frac{3}{4} \widetilde{K}_{3}-\frac{1}{2} \tilde{p}_{3},
$$

we obtain:

$$
\begin{aligned}
{ }_{1}\left\langle K K_{1}^{\prime} p_{1}^{\prime}\left|T^{(1)}\right| k\right\rangle= & {\left[\cos p_{1}^{\prime} / D\left(E, K_{1}^{\prime}\right)\right] } \\
& \times\left(-\frac{4 J}{N} \sum_{i}\left(\cos p_{l}-\cos \frac{1}{2} K_{i}\right) \delta\left(K_{1}^{\prime}-K_{i}\right)\right. \\
& -\frac{2 J}{N} \sum_{i=2}^{3} \sum_{\widetilde{K}_{i} \tilde{p}_{i}}\left(\cos \tilde{p}_{1}^{(i)}-\cos \frac{1}{2} \tilde{K}_{1}^{(i)}\right) \delta\left(K_{1}^{\prime}-\tilde{K}_{1}^{(i)}\right) \\
& \left.\times \frac{{ }_{i}\left\langle K \widetilde{K}_{i} \tilde{p}_{i}\left|T^{(i)}\right| \boldsymbol{k}\right\rangle}{E-E\left(\widetilde{K}_{i}, \tilde{p}_{i}\right)}\right),
\end{aligned}
$$

where we have substituted (B.10). Clearly ${ }_{1}\left\langle K K_{1}^{\prime} p_{1}^{\prime}\left|T^{(1)}\right| \boldsymbol{k}\right\rangle$ has the structure $\cos p_{1}^{\prime} \Phi\left(K_{1}^{\prime}, E\right)$ ( $\Phi$ being the same function of $K_{m}^{\prime}$ for all $\left.T^{(m)}, m=1,2,3\right)$.

We substitute this and (B.12) in the above equation, perform the sums of $\tilde{p}_{2}$ and $\tilde{p}_{3}$ using $\delta\left(K_{1}^{\prime}-\widetilde{K}_{1}^{(2)}\right)$ and $\delta\left(K_{1}^{\prime}-\widetilde{K}_{1}^{(3)}\right)$ and notice that both sums of $\tilde{K}_{2}$ and $\widetilde{K}_{3}$ are identical. This gives the following integral equation for $\Phi$ (the limit $N \rightarrow \infty$ has been taken):

$$
\begin{aligned}
\Phi\left(K_{1}^{\prime}, E\right)= & -\frac{2 J}{\pi D\left(E, K_{1}^{\prime}\right)}\left(\sum_{i=1}^{3}\left(\cos p_{i}-\cos \frac{1}{2} K_{i}\right) \delta\left(K_{1}^{\prime}-K_{i}\right)+\int_{-\pi}^{+\pi} \mathrm{d} \widetilde{K}_{2}\right. \\
& \left.\times \frac{\left[\cos \left(K-\widetilde{K}_{2}-\frac{1}{2} K_{1}^{\prime}\right)-\cos \frac{1}{2} K_{1}^{\prime}\right] \cos \left(K_{1}^{\prime}-K+\frac{1}{2} \tilde{K}_{2}\right)}{E-E\left(\widetilde{K}_{2}, K_{1}^{\prime}-K+\frac{1}{2} \widetilde{K}_{2}\right)} \Phi\left(\widetilde{K}_{2}, E\right)\right),
\end{aligned}
$$

with:

$$
\begin{aligned}
D\left(E, K_{1}^{\prime}\right)= & \frac{1}{\cos ^{2} \frac{1}{2} K_{1}^{\prime}} \\
& \times\left[t\left(1-\frac{\cos ^{2} \frac{1}{2} K_{1}^{\prime}}{t^{2}}\right)^{\frac{1}{2}}-t+\cos ^{2} \frac{1}{2} K_{1}^{\prime}\right]\left(1-\frac{\cos ^{2} \frac{1}{2} K_{1}^{\prime}}{t^{2}}\right)^{-\frac{1}{2}},
\end{aligned}
$$




$$
\begin{aligned}
t \equiv \frac{3}{2}-\frac{1}{2} \cos \left(K-K_{1}^{\prime}\right)-E / 2 J & \\
E-E\left(\tilde{K}_{2}, K_{1}^{\prime}-K+\frac{1}{2} \tilde{K}_{2}\right)= & E-3 J \\
& +J\left(\cos \left(K-\widetilde{K}_{2}\right)+\cos \left(K-K_{1}^{\prime}\right)\right. \\
& \left.+\cos \left(K-K_{1}^{\prime}-\widetilde{K}_{2}\right)\right) \\
= & 2 J \cos \frac{1}{2} \widetilde{K}_{2} \cos \left(K-K_{1}^{\prime}-\frac{1}{2} \widetilde{K}_{2}\right) \\
& +E-3 J+J \cos \left(K-\tilde{K}_{2}\right) \\
= & 2 J \cos \frac{1}{2} K_{1}^{\prime} \cos \left(K-\widetilde{K}_{2}-\frac{1}{2} K_{1}^{\prime}\right)-2 J t .
\end{aligned}
$$

\section{APPENDIX C}

We list here the explicit form of the three-body $T$ matrix in terms of the amplitudes $a$ and the resulting function $F_{\mathrm{p}}$ of eq. (6.14). In terms of Bethe-type, specialtype and extra-type amplitudes $\left\langle\boldsymbol{k}|V| \psi_{\mathbf{p}}\right\rangle$ is given by:

$$
\begin{aligned}
& \left\langle\boldsymbol{k}|\boldsymbol{V}| \psi_{\mathbf{p}}\right\rangle=-3 \sum_{\substack{m_{1} m_{2} \\
m_{1}<m_{2}-1}} a_{m_{1} m_{2}-1 m_{2}}^{\mathbf{B}}\left\langle\boldsymbol{k} \mid m_{1} m_{2}-1 m_{2}\right\rangle \\
& -3 \sum_{\substack{m_{1} m_{2} \\
m_{1}<m_{2}}} a_{m_{1} m_{2} m_{2}+1}^{\mathbf{B}}\left\langle\boldsymbol{k} \mid m_{1} m_{2} m_{2}+1\right\rangle \\
& -3 \sum_{\substack{m_{1} m_{2} \\
m_{1}>m_{2}}} a_{m_{2}-1 m_{2} m_{1}}^{\mathbf{B}}\left\langle\boldsymbol{k} \mid m_{2}-1 m_{2} m_{1}\right\rangle \\
& -3 \sum_{\substack{m_{1} m_{2} \\
m_{1}>m_{2}+1}} a_{m_{2} m_{2}+1 m_{1}}^{\mathbf{B}}\left\langle\boldsymbol{k} \mid m_{2} m_{2}+1 m_{1}\right\rangle \\
& -3 \sum_{m_{2}} a_{m_{2}-1 m_{2} m_{2}}^{\mathrm{s}}\left\langle\boldsymbol{k} \mid m_{2}-1 m_{2} m_{2}\right\rangle \\
& -3 \sum_{m_{2}} a_{m_{2} m_{2} m_{2}+1}^{\mathrm{s}}\left\langle\boldsymbol{k} \mid m_{2} m_{2} m_{2}+1\right\rangle \\
& -3 \sum_{m_{2}} a_{m_{2}-1 m_{2}-1 m_{2}}^{\mathrm{s}}\left\langle\boldsymbol{k} \mid m_{2}-1 m_{2}-1 m_{2}\right\rangle \\
& -3 \sum_{m_{2}} a_{m_{2} m_{2}+1 m_{2}+1}^{\mathrm{s}}\left\langle\boldsymbol{k} \mid m_{2} m_{2}+1 m_{2}+1\right\rangle \\
& +3 \sum_{\substack{m_{1} m_{2} \\
m_{1}>m_{2}}} \sum_{\Delta} a_{m_{2} m_{2} m_{1}}^{\mathbf{s}}\left\langle\boldsymbol{k} \mid m_{2} m_{2}+\Delta m_{1}\right\rangle
\end{aligned}
$$




$$
\begin{aligned}
& +3 \sum_{\substack{m_{1} m_{2} \\
m_{2}>m_{1}}} \sum_{\Delta} a_{m_{1} m_{2} m_{2}}^{\mathbf{S}}\left\langle\boldsymbol{k} \mid m_{1} m_{2} m_{2}+\Delta\right\rangle \\
& +3 \sum_{\substack{m_{1} m_{2} \\
m_{1} \backslash m_{2}}} \sum_{\Delta} a_{m_{1} m_{2} m_{2}}^{\mathrm{E}}\left\langle\boldsymbol{k} \mid m_{1} m_{2} m_{2}+\Delta\right\rangle \\
& +3 \sum_{\substack{m_{1} m_{2} \\
m_{1}>m_{2}}} \sum_{\Delta} a_{m_{2} m_{2} m_{1}}^{\mathbf{E}}\left\langle\boldsymbol{k} \mid m_{2} m_{2}+\Delta m_{1}\right\rangle \\
& +3 \sum_{m_{2}} \sum_{\Delta} a_{m_{2} m_{2} m_{2}}^{\mathbf{E}}\left\langle\boldsymbol{k} \mid m_{2} m_{2} m_{2}+\Delta\right\rangle \\
& -3 \sum_{m_{2}} \sum_{\Delta} a_{m_{2} m_{2} m_{2}+\Delta}^{\mathbf{E}}\left\langle\boldsymbol{k} \mid m_{2} m_{2} m_{2}+\Delta\right\rangle \\
& -3 \sum_{m_{2}} \sum_{\Delta} a_{m_{2} m_{2}+\Delta m_{2}+\Delta}^{\mathbf{E}}\left\langle\boldsymbol{k} \mid m_{2} m_{2}+\Delta m_{2}+\Delta\right\rangle .
\end{aligned}
$$

In order to write down explicitly the form of $F_{\mathrm{p}}$ present in eq. (6.14) let us define the following quantities:

1) $K$ is the total momentum of the three magnons. $k_{1}^{\prime}=u+i v, k_{2}^{\prime}=K-2 u$, $k_{3}^{\prime}=u-\mathrm{i} v$ are the momenta of the individual magnons in the bound state $\left|\psi_{\mathrm{p}}\right\rangle \cdot u$ and $v$ are determined by $K$ through the following equations:

$$
\begin{aligned}
& z=3 \operatorname{cotg} K ; \quad u=\operatorname{arccotg}(z / 3)-\operatorname{arccotg} z \quad(u>0) \\
& e^{-2 v}=\left(1+z^{2}\right) /\left(9+z^{2}\right) \quad(v>0) .
\end{aligned}
$$

2) $K_{1}=k_{2}+k_{3} ; p_{1}=\frac{1}{2}\left(k_{2}-k_{3}\right) ; k_{1}, k_{2}$ and $k_{3}$ are the momenta of the magnons in the bra.

3) $a=E-3, \quad b=E-1, \quad h(a)=\left(a^{2}-1\right)^{\frac{1}{2}}-a$,

$$
\Gamma(a)=\frac{1}{2} a+\frac{1}{2}\left(a^{2}-1\right)^{\frac{1}{2}}, \quad e^{r(a)}=\left|a+\left(a^{2}-1\right)^{\frac{1}{2}}\right|,
$$

$\operatorname{det} A=(b-\Gamma)\left[a(b-\Gamma)+\frac{3}{2}\right]$

4) $N(K, u, v)=\frac{1}{2}[\cos 2 u+\cosh 2 v]$

$$
+2 \cos (K-2 u) \cos u \cosh v+\cos ^{2}(K-2 u),
$$

$$
\begin{aligned}
R_{1}(K, u, v)= & \left\{2 \mathrm{e}^{-2 v}[\cos (K-2 u)+\cos u \cosh v]\right. \\
& +2 \mathrm{e}^{-v}[\cos (K-2 u) \cos (K-u)+\cos u \cos (K-u) \cosh v \\
& +\sin u \sin (K-u) \sinh v]\}[N(K, u, v)]^{-1}
\end{aligned}
$$




$$
\begin{aligned}
R_{2}(K, u, v) \\
=\left\{2 \mathrm{e}^{-3 v}\left[\cos u \cos (K-2 u)+\cos ^{2} u \cosh v+\sin ^{2} u \sinh v\right]\right. \\
\quad+2 \mathrm{e}^{-2 v}[\cos K \cos (K-2 u) \\
\quad+\cos K \cos u \cosh v+\sin K \sin u \sinh v]\} / N(K, u, v),
\end{aligned}
$$$$
I_{1}(K, u, v)=\left\{-2 \mathrm{e}^{-2 v} \sin u \sinh v\right.
$$$$
+2 \mathrm{e}^{-v}[\cos (K-2 u) \sin (K-u)+\cos u \sin (K-u) \cosh v
$$$$
-\sin u \cos (K-u) \sinh v]\} / N(K, u, v) \text {, }
$$

$I_{2}(K, u, v)=\left\{2 \mathrm{e}^{-3 v}[\sin u \cos (K-2 u)+\cos u \sin u \cosh v\right.$

$-\sin u \cos u \sinh v]+2 \mathrm{e}^{-2 v}[\sin K \cos (K-2 u)$

$+\sin K \cos u \cosh v-\cos K \sin u \sinh v]\} / N(K, u, v)$,

$$
\begin{aligned}
& \tilde{\alpha}+\bar{\gamma}=-2 \mathrm{e}^{-2 v}-b R_{1}(K, u, v)-\frac{1}{2} R_{2}(K, u, v), \\
& -\mathrm{i}(\bar{\alpha}-\bar{\gamma})=b I_{1}(K, u, v)+\frac{1}{2} I_{2}(K, u, v), \\
& \bar{\beta}=-\frac{3}{2} R_{1}(K, u, v), \\
& C_{N-2}+C_{N}=[(b-\Gamma) / \operatorname{det} A][a(\bar{\alpha}+\bar{y})+\bar{\beta}], \\
& -\mathrm{i}\left(C_{N-2}-C_{N}\right)=[-\mathrm{i}(\bar{\alpha}-\bar{\gamma}) / \operatorname{det} A]\left[a(b-\Gamma)+\frac{3}{2}\right], \\
& C_{N-1}=(1 / \operatorname{det} A)\left[-\frac{3}{2}(b-\Gamma)(\bar{\alpha}+\bar{\gamma})+(b-\Gamma)^{2} \tilde{\beta}\right], \\
& M_{+}=\frac{\cos \left(K-K_{1}\right)-\mathrm{e}^{f(a)}}{\cosh f(a)-\cos \left(K-K_{1}\right)} \\
& M_{-}=\frac{-\sin \left(K-K_{1}\right)}{\cosh f(a)-\cos \left(K-K_{1}\right)}, \\
& C_{+}=C_{N-2}+C_{N} ; \quad C_{-}=-\mathrm{i}\left(C_{N-2}-C_{N}\right), \\
& M_{+}=M_{1}+M_{2} ; \quad M_{-}=+\mathrm{i}\left(M_{1}-M_{2}\right) \text {, } \\
& F_{1}(K, u, v)=\left\{\cos ^{2}(K-2 u)+\cos u \cos (K-2 u) \cosh v\right. \\
& +\sin u \sin (K-2 u) \sinh v+\mathrm{e}^{-v}[\cos (K-2 u) \cos u \\
& \left.\left.+\cos ^{2} u \cosh v-\sin ^{2} u \sinh v\right]\right\} / N(K, u, v),
\end{aligned}
$$




$$
\begin{aligned}
F_{2}(K, u, v)= & -\{-\sin (K-2 u) \cos (K-2 u) \\
& -\cos u \sin (K-2 u) \cosh v+\sin u \cos (K-2 u) \sinh v \\
& +\mathrm{e}^{-v}[\sin u \cos (K-2 u)+\cos u \sin u \cosh v \\
& +\sin u \cos u \sinh v]\} / N(K, u, v)
\end{aligned}
$$

$$
\begin{aligned}
\Delta\left(K, u, v, a ; K_{1}\right)= & \cos \frac{1}{2} K_{1}\left(C_{N-1}-C_{+}\right)+\sin \frac{1}{2} K_{1} C_{-} \\
\Gamma\left(K, u, v, a ; K_{1}\right)= & -\left\{2 \mathrm { e } ^ { - v } \left[\cos \left(u-K+\frac{1}{2} K_{1}\right) \cos (K-2 u)\right.\right. \\
& +\cos u \cos \left(u-K+\frac{1}{2} K_{1}\right) \cosh v \\
& \left.-\sin u \sin \left(u-K+\frac{1}{2} K_{1}\right) \sinh v\right] \\
& +2 \mathrm{e}^{-2 v}\left[\cos \frac{1}{2} K_{1} \cos (K-2 u)+\cos \frac{1}{2} K_{1} \cos u \cosh v\right. \\
& \left.\left.-\sin \frac{1}{2} K_{1} \sin u \sinh v\right]\right\} N(K, u, v) .
\end{aligned}
$$

Then the expression for $F_{\mathrm{p}}\left(K_{1} ; K, u, v, a\right)$ can be written as:

$$
\begin{aligned}
F_{\mathbf{p}}\left(K_{1} ; K, u, v, a\right)= & -\left[a+\cos \left(K-K_{1}\right)\right] \\
& \times\left\{-\mathrm{e}^{-v} \cos \left(K-\frac{3}{2} K_{1}\right)+\mathrm{e}^{-2 v} \cos \left(u-\frac{1}{2} K_{1}\right)\right. \\
& +F_{1}(K, u, v) \cos \frac{1}{2} K_{1}\left[\cos \left(K-K_{1}-u\right)-\mathrm{e}^{v}\right] \\
& \left.+F_{2}(K, u, v) \cos \frac{1}{2} K_{1} \sin \left(K-K_{1}-u\right)\right\} \\
& +\frac{1}{2} \cos \frac{1}{2} K_{1} h(a)\left[\cosh v-\cos \left(K-K_{1}-u\right)\right] \\
& \times\left\{C_{+}\left[\cos \left(K-K_{1}\right)-\mathrm{e}^{f(a)}\right]-C_{-} \sin \left(K-K_{1}\right)\right\} \\
& +\left[a+\cos \left(K-K_{1}\right)\right]\left[\cos \left(K-K_{1}-u\right)-\cosh v\right] \\
& \times\left[\Gamma\left(K, u, v ; K_{1}\right)+\Delta\left(K, u, v ; K_{1}\right)\right] .
\end{aligned}
$$

Although this expression looks highly complicated it has the very simple mathematical property that it is analytic in the variable $z=\mathrm{e}^{\mathrm{i} K_{1}}$ except for poles at $z=0$ and $\infty$.

\section{REFERENCES}

1) Bethe, H., Z. Physik 71 (1931) 205.

2) Dyson, F.J., Phys. Rev. 102 (1956) 1217.

3) Maleev, S., Soviet Physics-JETP 6 (1958) 776.

4) Faddeev, L., Soviet Physics-JETP 12 (1961) 1014.

5) Dodd, L.R., J. math. Phys. 11 (1970) 207.

6) Majumdar, C.K., Phys. Rev. B1 (1970) 287.

7) Majumdar, C.K. and Mukhopadhyay, G., Phys. Letters 31A (1970) 321.

8) Orbach, R., Phys. Rev. 112 (1958) 309.

9) Gochev, I. G., Soviet Physics-JETP 34 (1972) 892. 